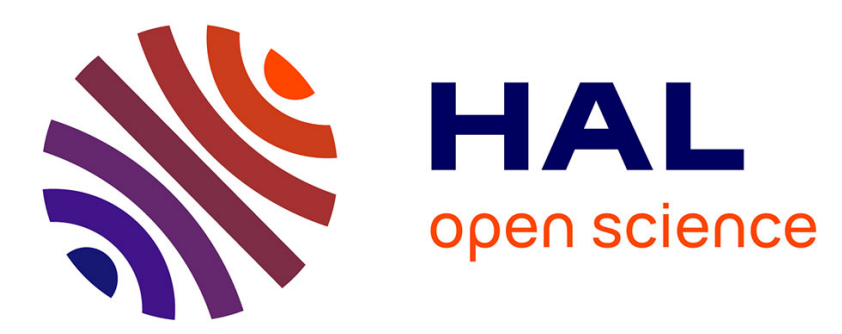

\title{
Finite Larmor radius approximation for collisional magnetic confinement. Part I: The linear Boltzmann equation
}

Mihai Bostan, Céline Caldini Queiros

\section{- To cite this version:}

Mihai Bostan, Céline Caldini Queiros. Finite Larmor radius approximation for collisional magnetic confinement. Part I: The linear Boltzmann equation. Quarterly journal of pure and applied mathematics, 2014, LXXII (2), pp.323-345. 10.1090/S0033-569X-2014-01356-1 . hal-01266550

\section{HAL Id: hal-01266550 \\ https://hal.science/hal-01266550}

Submitted on 2 Feb 2016

HAL is a multi-disciplinary open access archive for the deposit and dissemination of scientific research documents, whether they are published or not. The documents may come from teaching and research institutions in France or abroad, or from public or private research centers.
L'archive ouverte pluridisciplinaire HAL, est destinée au dépôt et à la diffusion de documents scientifiques de niveau recherche, publiés ou non, émanant des établissements d'enseignement et de recherche français ou étrangers, des laboratoires publics ou privés. 


\title{
FINITE LARMOR RADIUS APPROXIMATION FOR COLLISIONAL MAGNETIC CONFINEMENT. PART I: THE LINEAR BOLTZMANN EQUATION
}

\author{
BY
}

MIHAI BOSTAN (Laboratoire d'Analyse, Topologie, Probabilités LATP, Centre de Mathématiques et Informatique CMI, UMR CNRS 7353, 39 rue Frédéric Joliot Curie, 13453 Marseille Cedex 13 France)

AND

CÉLINE CALDINI-QUEIROS (Laboratoire de Mathématiques de Besançon, UMR CNRS 6623, Université de Franche-Comté, 16 route de Gray, 25030 Besançon Cedex France)

Abstract. The subject matter of this paper concerns the derivation of the finite Larmor radius approximation, when collisions are taken into account. Several studies are performed, corresponding to different collision kernels : the relaxation and the FokkerPlanck operators. Gyroaveraging the relaxation operator leads to a position-velocity integral operator, whereas gyroaveraging the linear Fokker-Planck operator leads to diffusion in velocity but also with respect to the perpendicular position coordinates.

\section{Introduction.}

Many studies in plasma physics concern the energy production through thermonuclear fusion. In particular this reaction can be achieved by magnetic confinement i.e., a tokamak plasma is controlled by applying a strong magnetic field. Large magnetic fields induce high cyclotronic frequencies corresponding to the fast particle dynamics around the magnetic lines. We concentrate on the linear problem, by neglecting the

2000 Mathematics Subject Classification. Primary 35Q75, 78A35, 82D10.

E-mail address: bostan@cmi.univ-mrs.fr

E-mail address: celine.caldini-queiros@univ-fcomte.fr 
self-consistent electro-magnetic field. The external electro-magnetic field is supposed to be a given smooth field

$$
E=-\nabla_{x} \phi, \quad B^{\varepsilon}=\frac{B(x)}{\varepsilon} b(x), \quad|b|=1
$$

when $\varepsilon>0$ is a small parameter, destinated to converge to 0 , in order to describe strong magnetic fields. The scalar function $\phi$ stands for the electric potential, $B(x)>0$ is the rescaled magnitude of the magnetic field and $b(x)$ denotes its direction. As usual, we appeal to the kinetic description for studying the evolution of the plasma. The notation $f^{\varepsilon}=f^{\varepsilon}(t, x, v) \geq 0$ stands for the presence density of a population of charged particles with mass $m$ and charge $q$. This density satisfies

$$
\begin{gathered}
\partial_{t} f^{\varepsilon}+v \cdot \nabla_{x} f^{\varepsilon}+\frac{q}{m}\left(E+v \wedge B^{\varepsilon}\right) \cdot \nabla_{v} f^{\varepsilon}=Q\left(f^{\varepsilon}\right), \quad(t, x, v) \in \mathbb{R}_{+} \times \mathbb{R}^{3} \times \mathbb{R}^{3} \\
f^{\varepsilon}(0, x, v)=f^{\text {in }}(x, v), \quad(x, v) \in \mathbb{R}^{3} \times \mathbb{R}^{3}
\end{gathered}
$$

where $Q$ denotes a collision kernel. The interpretation of the density $f^{\varepsilon}$ is straightforward : the number of charged particles contained at time $t$ inside the infinitesimal volume $\mathrm{d} x \mathrm{~d} v$ around the point $(x, v)$ of the position-velocity phase space is given by $f^{\varepsilon}(t, x, v) \mathrm{d} x \mathrm{~d} v$. The equation (1.1) accounts for the fluctuation of the density $f^{\varepsilon}$ due to the transport but also to the collisions. We analyze here the linear relaxation operator. The bilinear Fokker-Planck-Landau operator will be studied in [6] following similar lines.

When neglecting the collisions the limit model as $\varepsilon \searrow 0$ comes by averaging with respect to the fast cyclotronic motion $[15,19,10,1,2,3,4]$. The problem reduces to homogenization analysis and can be solved using the notion of two-scale convergence $[12,13,11]$.

We point out that a linearized and gyroaveraged collision operator has been written in [20], but the implementation of this operator seems very hard. We refer to [8, 9] for a general guiding-center bilinear Fokker-Planck collision operator. Another difficulty lies in the relaxation of the distribution function towards a local Maxwellian equilibrium. Most of the available model operators, in particular those which are linearized near a Maxwellian, are missing this property. Very recently a set of model collision operators has been obtained in [14], based on entropy variational principles [7]. 
We study here the finite Larmor radius scaling i.e., the typical perpendicular spatial length is of the same order as the Larmor radius and the parallel spatial length is much larger. We assume that the magnetic field is homogeneous and stationary

$$
B^{\varepsilon}=\left(0,0, \frac{B}{\varepsilon}\right)
$$

for some constant $B>0$ and therefore (1.1) becomes

$\partial_{t} f^{\varepsilon}+\frac{1}{\varepsilon}\left(v_{1} \partial_{x_{1}} f^{\varepsilon}+v_{2} \partial_{x_{2}} f^{\varepsilon}\right)+v_{3} \partial_{x_{3}} f^{\varepsilon}+\frac{q}{m} E \cdot \nabla_{v} f^{\varepsilon}+\frac{\omega_{c}}{\varepsilon}\left(v_{2} \partial_{v_{1}} f^{\varepsilon}-v_{1} \partial_{v_{2}} f^{\varepsilon}\right)=Q\left(f^{\varepsilon}\right)$

where $\omega_{c}=q B / m$ stands for the rescaled cyclotronic frequency. The density $f^{\varepsilon}$ is decomposed into a dominant density $f$ and fluctuations of orders $\varepsilon, \varepsilon^{2}, \ldots$

$$
f^{\varepsilon}=f+\varepsilon f^{1}+\varepsilon^{2} f^{2}+\ldots
$$

Combining (1.3), (1.4) yields, with the notations $\bar{x}=\left(x_{1}, x_{2}\right), \bar{v}=\left(v_{1}, v_{2}\right),{ }^{\perp} \bar{v}=$ $\left(v_{2},-v_{1}\right)$

$$
\begin{gathered}
\mathcal{T} f:=\bar{v} \cdot \nabla_{\bar{x}} f+\omega_{c}{ }^{\perp} \bar{v} \cdot \nabla_{\bar{v}} f=0 \\
\partial_{t} f+v_{3} \partial_{x_{3}} f+\frac{q}{m} E \cdot \nabla_{v} f+\mathcal{T} f^{1}=Q(f)
\end{gathered}
$$

The equation (1.5) appears as a divergence constraint

$$
\operatorname{div}_{x, v}\left\{f\left(\bar{v}, 0, \omega_{c}{ }^{\perp} \bar{v}, 0\right)\right\}=0 .
$$

Equivalently, (1.5) says that at any time $t$ the density $f(t, \cdot, \cdot)$ remains constant along the flow associated to $\bar{v} \cdot \nabla_{\bar{x}}+\omega_{c}{ }^{\perp} \bar{v} \cdot \nabla_{\bar{v}}$

$$
\frac{\mathrm{d} \bar{X}}{\mathrm{~d} s}=\bar{V}(s), \quad \frac{\mathrm{d} X_{3}}{\mathrm{~d} s}=0, \quad \frac{\mathrm{d} \bar{V}}{\mathrm{~d} s}=\omega_{c}{ }^{\perp} \bar{V}(s), \quad \frac{\mathrm{d} V_{3}}{\mathrm{~d} s}=0
$$

and therefore, at any time $t$, the density $f(t, \cdot, \cdot)$ depends only on the invariants of (1.7)

$$
f(t, x, v)=g\left(t, x_{1}+\frac{v_{2}}{\omega_{c}}, x_{2}-\frac{v_{1}}{\omega_{c}}, x_{3}, r=|\bar{v}|, v_{3}\right) .
$$

The time evolution for $f$ comes by eliminating $f^{1}$ in (1.6). For doing that, we project onto the kernel of $\mathcal{T}$, which is orthogonal to the range of $\mathcal{T}$. In order to get a explicit model for $f$ we need a simpler representation for the orthogonal projection on $\operatorname{ker} \mathcal{T}$. Actually 
this projection appears as the average along the characteristic flow (1.7). Denoting by $\langle\cdot\rangle$ this projection, we obtain

$$
\left\langle\partial_{t} f+v_{3} \partial_{x_{3}} f+\frac{q}{m} E \cdot \nabla_{v} f\right\rangle=\langle Q(f)\rangle, \quad(t, x, v) \in \mathbb{R}_{+} \times \mathbb{R}^{3} \times \mathbb{R}^{3} .
$$

By one hand, averaging $\partial_{t}+v_{3} \partial_{x_{3}}+\frac{q}{m} E \cdot \nabla_{v}$ leads to another transport operator

$$
\left\langle\partial_{t} f+v_{3} \partial_{x_{3}} f+\frac{q}{m} E \cdot \nabla_{v} f\right\rangle=\partial_{t} f+\frac{\langle\perp \bar{E}\rangle}{B} \cdot \nabla_{\bar{x}} f+v_{3} \partial_{x_{3}} f+\frac{q}{m}\left\langle E_{3}\right\rangle \partial_{v_{3}} f .
$$

The key point here is to choose as new coordinates the invariants of (1.7) and to observe that the partial derivatives with respect to these invariants commute with the average operator. More generally, for any smooth vector field $\xi=\left(\xi_{x}, \xi_{v}\right)$, we obtain the following commutation formula between the divergence and average operators, cf. Proposition 3.3

$$
\begin{aligned}
\left\langle\operatorname{div}_{x, v} \xi\right\rangle & =\operatorname{div}_{\bar{x}}\left\{\left\langle\xi_{\bar{x}}+\frac{{ }^{\perp} \xi_{\bar{v}}}{\omega_{c}}\right\rangle+\left\langle\xi_{\bar{v}} \cdot \frac{{ }^{\perp} \bar{v}}{|\bar{v}|}\right\rangle \frac{\bar{v}}{\omega_{c}|\bar{v}|}-\left\langle\xi_{\bar{v}} \cdot \frac{\bar{v}}{|\bar{v}|}\right\rangle \frac{{ }^{\perp} \bar{v}}{\omega_{c}|\bar{v}|}\right\}+\partial_{x_{3}}\left\langle\xi_{x_{3}}\right\rangle \\
& +\operatorname{div}_{\bar{v}}\left\{\left\langle\xi_{\bar{v}} \cdot \frac{{ }^{\perp} \bar{v}}{|\bar{v}|}\right\rangle \frac{{ }^{\perp} \bar{v}}{|\bar{v}|}+\left\langle\xi_{\bar{v}} \cdot \frac{\bar{v}}{|\bar{v}|}\right\rangle \frac{\bar{v}}{|\bar{v}|}\right\}+\partial_{v_{3}}\left\langle\xi_{v_{3}}\right\rangle .
\end{aligned}
$$

By the other hand we need to compute the average of the collision kernel $Q$ which is a more complicated task. We focus on the relaxation Boltzmann operator [16]

$$
Q_{B}(f(t, x, \cdot))(v)=\frac{1}{\tau} \int_{\mathbb{R}^{3}} s\left(v, v^{\prime}\right)\left\{M(v) f\left(t, x, v^{\prime}\right)-M\left(v^{\prime}\right) f(t, x, v)\right\} \mathrm{d} v^{\prime}
$$

where $\tau>0$ is the relaxation time, $s\left(v, v^{\prime}\right)$ is the scattering cross section and $M$ is the Maxwellian equilibrium with temperature $\theta$

$$
M(v)=\frac{1}{(2 \pi \theta / m)^{3 / 2}} e^{-\frac{m|v|^{2}}{2 \theta}}, \quad v \in \mathbb{R}^{3} .
$$

We need to average functions like $(x, v) \rightarrow \int_{\mathbb{R}^{3}} C\left(v, v^{\prime}\right) f\left(x, v^{\prime}\right) \mathrm{d} v^{\prime}$, where $C\left(v, v^{\prime}\right)$ is a given function. Since the invariants of the flow $(\bar{X}, \bar{V})$ combines $\bar{x}$ and $\bar{v}$, we get a position-velocity integral operator cf. Proposition 4.2

$$
\left\langle\int_{\mathbb{R}^{3}} C\left(v, v^{\prime}\right) f\left(x, v^{\prime}\right) \mathrm{d} v^{\prime}\right\rangle(x, v)=\omega_{c}^{2} \int_{\mathbb{R}^{2}} \int_{\mathbb{R}^{3}} \mathcal{C}\left(|\bar{v}|, v_{3},\left|\overline{v^{\prime}}\right|, v_{3}^{\prime}, z\right) f\left(\overline{x^{\prime}}, x_{3}, v^{\prime}\right) \mathrm{d} v^{\prime} \mathrm{d} x_{1}^{\prime} \mathrm{d} x_{2}^{\prime}
$$


with $z=\omega_{c} \bar{x}+{ }^{\perp} \bar{v}-\left(\omega_{c} \overline{x^{\prime}}+{ }^{\perp} \overline{v^{\prime}}\right)$. We prove that averaging $Q_{B}$ will lead to a positionvelocity integral operator of the same form

$\left\langle Q_{B}\right\rangle f(x, v):=\left\langle Q_{B}(f)\right\rangle(x, v)$

$$
=\frac{\omega_{c}^{2}}{\tau} \int_{\mathbb{R}^{2}} \int_{\mathbb{R}^{3}} \mathcal{S}\left(|\bar{v}|, v_{3},\left|\overline{v^{\prime}}\right|, v_{3}^{\prime}, z\right)\left\{M(v) f\left(\overline{x^{\prime}}, x_{3}, v^{\prime}\right)-M\left(v^{\prime}\right) f(x, v)\right\} \mathrm{d} v^{\prime} \mathrm{d} x_{1}^{\prime} \mathrm{d} x_{2}^{\prime}
$$

(see Theorem 1.1 for the definition of $\mathcal{S}$ ). Observe that $\left\langle Q_{B}\right\rangle$ is global in $(\bar{x}, v)$, but remains local in $x_{3}$. In particular it satisfies only a global mass balance, which comes easily by Fubini theorem and the symmetry of $\mathcal{S}$ cf. Remark 4.6

$$
\int_{\mathbb{R}^{3}} \int_{\mathbb{R}^{3}}\left\langle Q_{B}\right\rangle f(x, v) \mathrm{d} v \mathrm{~d} x=0 .
$$

In the case of the relaxation operator $Q_{B}$ we obtain the limit model

Theorem 1.1. Assume that the scattering cross section satisfies (4.2), (4.7) and that $E(x)=-\nabla_{x} \phi(x), \phi \in W^{2, \infty}\left(\mathbb{R}^{3}\right)$. Let us consider $f^{\text {in }} \geq 0, f^{\text {in }} \in L^{1}\left(\mathbb{R}^{3} \times \mathbb{R}^{3}\right) \cap$ $L^{2}\left(M^{-1} \mathrm{~d} x \mathrm{~d} v\right)$ and denote by $f^{\varepsilon}$ the weak solution of (1.3), (1.2) with $Q=Q_{B}$ for any $\varepsilon>0$. We assume that $\left(f^{\varepsilon}\right)_{\varepsilon>0}$ is bounded in $L^{\infty}\left(\mathbb{R}_{+}, L^{2}\left(M^{-1} \mathrm{~d} x \mathrm{~d} v\right)\right)$. Then the family $\left(f^{\varepsilon}\right)_{\varepsilon>0}$ converges weakly $\star$ in $L^{\infty}\left(\mathbb{R}_{+}, L^{2}\left(M^{-1} \mathrm{~d} x \mathrm{~d} v\right)\right)$ to the weak solution of

$$
\begin{gathered}
\partial_{t} f+\frac{\langle\perp \bar{E}\rangle}{B} \cdot \nabla_{\bar{x}} f+v_{3} \partial_{x_{3}} f+\frac{q}{m}\left\langle E_{3}\right\rangle \partial_{v_{3}} f=\left\langle Q_{B}\right\rangle f, \quad(t, x, v) \in \mathbb{R}_{+} \times \mathbb{R}^{3} \times \mathbb{R}^{3} \\
f(0, x, v)=\left\langle f^{\text {in }}\right\rangle(x, v), \quad(x, v) \in \mathbb{R}^{3} \times \mathbb{R}^{3}
\end{gathered}
$$

where the averaged relaxation operator is given by

$\left\langle Q_{B}\right\rangle f(x, v)=\frac{\omega_{c}^{2}}{\tau} \int_{\mathbb{R}^{2}} \int_{\mathbb{R}^{3}} \mathcal{S}\left(|\bar{v}|, v_{3},\left|\overline{v^{\prime}}\right|, v_{3}^{\prime}, z\right)\left\{M(v) f\left(\overline{x^{\prime}}, x_{3}, v^{\prime}\right)-M\left(v^{\prime}\right) f(x, v)\right\} \mathrm{d} v^{\prime} \mathrm{d} x_{1}^{\prime} \mathrm{d} x_{2}^{\prime}$

with $z=\omega_{c} \bar{x}+{ }^{\perp} \bar{v}-\left(\omega_{c} \overline{x^{\prime}}+{ }^{\perp} \overline{v^{\prime}}\right)$ and the averaged scattering cross section writes

$$
\mathcal{S}\left(r, v_{3}, r^{\prime}, v_{3}^{\prime}, z\right)=\sigma\left(\sqrt{|z|^{2}+\left(v_{3}-v_{3}^{\prime}\right)^{2}}\right) \chi\left(r, r^{\prime}, z\right)
$$

with

$$
\chi\left(r, r^{\prime}, z\right)=\frac{\mathbf{1}_{\left\{\left|r-r^{\prime}\right|<|z|<r+r^{\prime}\right\}}}{\pi^{2} \sqrt{|z|^{2}-\left(r-r^{\prime}\right)^{2}} \sqrt{\left(r+r^{\prime}\right)^{2}-|z|^{2}}}, \quad r, r^{\prime} \in \mathbb{R}_{+}, v_{3}, v_{3}^{\prime} \in \mathbb{R}, \quad z \in \mathbb{R}^{2} .
$$


The averaging technique allows us to treat many different collision operators, for example the Fokker-Planck kernel (see Appendix A for details)

$$
Q_{F P}(f)=\frac{\theta}{m \tau} \operatorname{div}_{v}\left(\nabla_{v} f+\frac{m}{\theta} v f\right)=\frac{\theta}{m \tau} \operatorname{div}_{v}\left\{M \nabla_{v}\left(\frac{f}{M}\right)\right\}
$$

Theorem 1.2. The limit model when $\varepsilon \searrow 0$ of (1.3), (1.2) with $Q=Q_{F P}$ is given by

$$
\begin{gathered}
\partial_{t} f+\frac{\langle\perp \bar{E}\rangle}{B} \cdot \nabla_{\bar{x}} f+v_{3} \partial_{x_{3}} f+\frac{q}{m}\left\langle E_{3}\right\rangle \partial_{v_{3}} f=\left\langle Q_{F P}\right\rangle f, \quad(t, x, v) \in \mathbb{R}_{+} \times \mathbb{R}^{3} \times \mathbb{R}^{3} \\
f(0, x, v)=\left\langle f^{\text {in }}\right\rangle(x, v), \quad(x, v) \in \mathbb{R}^{3} \times \mathbb{R}^{3}
\end{gathered}
$$

where the averaged Fokker-Planck operator and the diffusion matrix $\mathcal{L}$ write

$$
\begin{gathered}
\left\langle Q_{F P}\right\rangle f(x, v)=\frac{\theta}{m \tau} \operatorname{div}_{\omega_{c} x, v}\left\{M \mathcal{L} \nabla_{\omega_{c} x, v}\left(\frac{f}{M}\right)\right\} \\
\mathcal{L}=\left(\begin{array}{cc}
2\left(I_{3}-e_{3} \otimes e_{3}\right) & -E \\
E & I_{3}
\end{array}\right), E=\left(\begin{array}{rrr}
0 & 1 & 0 \\
-1 & 0 & 0 \\
0 & 0 & 0
\end{array}\right) .
\end{gathered}
$$

Notice that the averaged Fokker-Planck operator contains no derivatives with respect to $x_{3}$ since the diffusion matrix $\mathcal{L}$ has only zero entries on the third line and column; averaging the Fokker-Planck operator leads to diffusion in velocity but also with respect to the perpendicular position coordinates.

Our paper is organized as follows. In Section 2 we introduce the average operator along a characteristic flow. Section 3 is devoted to the commutation properties between average and first order differential operators. The average of the linear Boltzmann kernel is computed in Section 4. We establish its main properties and we prove the convergence result stated in Theorem 1.1 .

2. Average operator. We recall briefly the definition and properties of the average operator corresponding to the transport operator $\mathcal{T}$, whose definition in the $L^{2}\left(\mathbb{R}^{3} \times \mathbb{R}^{3}\right)$ setting is

$$
\mathcal{T} u=\operatorname{div}_{x, v}(u b), \quad b=\left(\bar{v}, 0, \omega_{c}^{\perp} \bar{v}, 0\right), \quad \omega_{c}=\frac{q B}{m}
$$


for any function $u$ in the domain

$$
\mathrm{D}(\mathcal{T})=\left\{u(x, v) \in L^{2}\left(\mathbb{R}^{3} \times \mathbb{R}^{3}\right): \operatorname{div}_{x, v}(u b) \in L^{2}\left(\mathbb{R}^{3} \times \mathbb{R}^{3}\right)\right\} .
$$

We denote by $\|\cdot\|$ the standard norm of $L^{2}\left(\mathbb{R}^{3} \times \mathbb{R}^{3}\right)$. The characteristics $(X, V)(s ; x, v)$ associated to $\bar{v} \cdot \nabla_{\bar{x}}+\omega_{c}{ }^{\perp} \bar{v} \cdot \nabla_{\bar{v}}$, see (1.7), satisfy

$$
\frac{\mathrm{d}}{\mathrm{d} s}\left\{\bar{X}+\frac{{ }^{\perp} \bar{V}}{\omega_{c}}\right\}=0, \quad \frac{\mathrm{d} \bar{V}}{\mathrm{~d} s}=\omega_{c}{ }^{\perp} \bar{V}, \quad \frac{\mathrm{d} X_{3}}{\mathrm{~d} s}=0, \quad \frac{\mathrm{d} V_{3}}{\mathrm{~d} s}=0
$$

implying that

$$
\bar{V}(s)=R\left(-\omega_{c} s\right) \bar{v}, \quad \bar{X}(s)=\bar{x}+\frac{\perp \bar{v}}{\omega_{c}}-\frac{\perp \bar{V}(s)}{\omega_{c}}, \quad X_{3}(s)=x_{3}, \quad V_{3}(s)=v_{3}
$$

where $R(\alpha)$ stands for the rotation of angle $\alpha$

$$
R(\alpha)=\left(\begin{array}{rr}
\cos \alpha & -\sin \alpha \\
\sin \alpha & \cos \alpha
\end{array}\right) .
$$

All the trajectories are $T_{c}=2 \pi / \omega_{c}$ periodic and we introduce the average operator, see [2], for any function $u \in L^{2}\left(\mathbb{R}^{3} \times \mathbb{R}^{3}\right)$

$$
\begin{aligned}
\langle u\rangle(x, v) & =\frac{1}{T_{c}} \int_{0}^{T_{c}} u(X(s ; x, v), V(s ; x, v)) \mathrm{d} s \\
& =\frac{1}{2 \pi} \int_{0}^{2 \pi} u\left(\bar{x}+\frac{\perp \bar{v}}{\omega_{c}}-\frac{\perp\{R(\alpha) \bar{v}\}}{\omega_{c}}, x_{3}, R(\alpha) \bar{v}, v_{3}\right) \mathrm{d} \alpha .
\end{aligned}
$$

It is convenient to introduce the notation $e^{i \varphi}$ for the $\mathbb{R}^{2}$ vector $(\cos \varphi, \sin \varphi)$. Assume that the vector $\bar{v}$ writes $\bar{v}=|\bar{v}| e^{i \varphi}$. Then $R(\alpha) \bar{v}=|\bar{v}| e^{i(\alpha+\varphi)}$ and the expression for $\langle u\rangle$ becomes

$$
\begin{aligned}
\langle u\rangle(x, v) & =\frac{1}{2 \pi} \int_{0}^{2 \pi} u\left(\bar{x}+\frac{{ }^{\perp} \bar{v}}{\omega_{c}}-\frac{\perp\left\{|\bar{v}| e^{i(\alpha+\varphi)}\right\}}{\omega_{c}}, x_{3},|\bar{v}| e^{i(\alpha+\varphi)}, v_{3}\right) \mathrm{d} \alpha \\
& =\frac{1}{2 \pi} \int_{0}^{2 \pi} u\left(\bar{x}+\frac{{ }^{\perp} \bar{v}}{\omega_{c}}-\frac{\perp\left\{|\bar{v}| e^{i \alpha}\right\}}{\omega_{c}}, x_{3},|\bar{v}| e^{i \alpha}, v_{3}\right) \mathrm{d} \alpha .
\end{aligned}
$$

Notice that $\langle u\rangle$ depends only on the invariants $\bar{x}+\frac{{ }^{\perp} \bar{v}}{\omega_{c}},|\bar{v}|, x_{3}, v_{3}$ and therefore belongs to ker $\mathcal{T}$. The following two results are justified in [3], Propositions 2.1, 2.2. The first one states that averaging reduces to orthogonal projection onto the kernel of $\mathcal{T}$. The second one concerns the invertibility of $\mathcal{T}$ on the subspace of zero average functions and establishes a Poincaré inequality. 
Proposition 2.1. The average operator is linear continuous. Moreover it coincides with the orthogonal projection on the kernel of $\mathcal{T}$ i.e.,

$$
\langle u\rangle \in \operatorname{ker} \mathcal{T} \text { and } \int_{\mathbb{R}^{3}} \int_{\mathbb{R}^{3}}(u-\langle u\rangle) \varphi \mathrm{d} v \mathrm{~d} x=0, \quad \forall \varphi \in \operatorname{ker} \mathcal{T}
$$

REMARK 2.1. Notice that $(\bar{X}, \bar{V})$ depends only on $s$ and $(\bar{x}, \bar{v})$ and thus the variational characterization in $(2.2)$ holds true at any fixed $\left(x_{3}, v_{3}\right) \in \mathbb{R}^{2}$. Indeed, for any $\varphi \in \operatorname{ker} \mathcal{T}$, $\left(x_{3}, v_{3}\right) \in \mathbb{R}^{2}$ we have

$$
\begin{aligned}
\int_{\mathbb{R}^{2}} \int_{\mathbb{R}^{2}}(u \varphi)(x, v) \mathrm{d} \bar{v} \mathrm{~d} \bar{x} & =\frac{1}{T_{c}} \int_{0}^{T_{c}} \int_{\mathbb{R}^{2}} \int_{\mathbb{R}^{2}} u(x, v) \varphi\left(\bar{X}(-s ; x, v), x_{3}, \bar{V}(-s ; x, v), v_{3}\right) \mathrm{d} \bar{v} \mathrm{~d} \bar{x} \mathrm{~d} s \\
& =\frac{1}{T_{c}} \int_{0}^{T_{c}} \int_{\mathbb{R}^{2}} \int_{\mathbb{R}^{2}} u\left(\bar{X}(s ; x, v), x_{3}, \bar{V}(s ; x, v), v_{3}\right) \varphi(x, v) \mathrm{d} \bar{v} \mathrm{~d} \bar{x} \mathrm{~d} s \\
& =\int_{\mathbb{R}^{2}} \int_{\mathbb{R}^{2}}\langle u\rangle(x, v) \varphi(x, v) \mathrm{d} \bar{v} \mathrm{~d} \bar{x} .
\end{aligned}
$$

We have the orthogonal decomposition of $L^{2}\left(\mathbb{R}^{3} \times \mathbb{R}^{3}\right)$ into invariant functions along the characteristics (1.7) and zero average functions

$$
u=\langle u\rangle+(u-\langle u\rangle), \quad \int_{\mathbb{R}^{3}} \int_{\mathbb{R}^{3}}(u-\langle u\rangle)\langle u\rangle \mathrm{d} v \mathrm{~d} x=0 .
$$

Notice that $\mathcal{T}^{\star}=-\mathcal{T}$ and thus the equality $\langle\cdot\rangle=\operatorname{Proj}_{\text {ker } \mathcal{T}}$ implies

$$
\operatorname{ker}\langle\cdot\rangle=(\operatorname{ker} \mathcal{T})^{\perp}=\left(\operatorname{ker} \mathcal{T}^{\star}\right)^{\perp}=\overline{\text { Range } \mathcal{T}}
$$

In particular Range $\mathcal{T} \subset \operatorname{ker}\langle\cdot\rangle$. Actually we show that Range $\mathcal{T}$ is closed, which will give a solvability condition for $\mathcal{T} u=w($ cf. [3], Propositions 2.2).

Proposition 2.2. The restriction of $\mathcal{T}$ to $\operatorname{ker}\langle\cdot\rangle$ is one to one map onto $\operatorname{ker}\langle\cdot\rangle$. Its inverse belongs to $\mathcal{L}(\operatorname{ker}\langle\cdot\rangle, \operatorname{ker}\langle\cdot\rangle)$ and we have the Poincaré inequality

$$
\|u\| \leq \frac{2 \pi}{\left|\omega_{c}\right|}\|\mathcal{T} u\|, \quad \omega_{c}=\frac{q B}{m} \neq 0
$$

for any $u \in \mathrm{D}(\mathcal{T}) \cap \operatorname{ker}\langle\cdot\rangle$.

The natural space when dealing with the linear Boltzmann kernel $Q_{B}$ is $L^{2}\left(M^{-1} \mathrm{~d} x \mathrm{~d} v\right)$ rather than $L^{2}(\mathrm{~d} x \mathrm{~d} v)$. Motivated by that we introduce the operator $\mathcal{T}_{M}: \mathrm{D}\left(\mathcal{T}_{M}\right) \subset$ 
$L^{2}\left(M^{-1} \mathrm{~d} x \mathrm{~d} v\right) \rightarrow L^{2}\left(M^{-1} \mathrm{~d} x \mathrm{~d} v\right)$ given by $\mathcal{T}_{M} u=\operatorname{div}_{x, v}(u b)$ for any function $u$ in the domain

$$
\mathrm{D}\left(\mathcal{T}_{M}\right)=\left\{u(x, v) \in L^{2}\left(M^{-1} \mathrm{~d} x \mathrm{~d} v\right): \operatorname{div}_{x, v}(u b) \in L^{2}\left(M^{-1} \mathrm{~d} x \mathrm{~d} v\right)\right\} .
$$

Straightforward arguments show that $u \in \mathrm{D}\left(\mathcal{T}_{M}\right)$ iff $u / \sqrt{M} \in \mathrm{D}(\mathcal{T})$ and $\mathcal{T}_{M}(u)=$ $\sqrt{M} \mathcal{T}(u / \sqrt{M})$ for any $u \in \mathcal{D}\left(\mathcal{T}_{M}\right)$. In particular we have ker $\mathcal{T}_{M}=\sqrt{M} \operatorname{ker} \mathcal{T}$. Notice that formula (2.1) still defines a linear bounded operator on $L^{2}\left(M^{-1} \mathrm{~d} x \mathrm{~d} v\right)$, denoted by $\langle\cdot\rangle_{M}$, which coincides with the orthogonal projection on the kernel of $\mathcal{T}_{M}$, with respect to the scalar product of $L^{2}\left(M^{-1} \mathrm{~d} x \mathrm{~d} v\right)$. Indeed, taking into account that $M(v)$ is constant along the characteristic flow of (1.7), we have for any $u \in L^{2}\left(M^{-1} \mathrm{~d} x \mathrm{~d} v\right)$

$$
\langle u\rangle_{M}=\sqrt{M}\left\langle\frac{u}{\sqrt{M}}\right\rangle \in \sqrt{M} \operatorname{ker} \mathcal{T}=\operatorname{ker} \mathcal{T}_{M}
$$

and for any $\varphi \in \operatorname{ker} \mathcal{T}_{M}$

$$
\int_{\mathbb{R}^{3}} \int_{\mathbb{R}^{3}}\left(u-\langle u\rangle_{M}\right) \varphi(x, v) \frac{\mathrm{d} x \mathrm{~d} v}{M(v)}=\int_{\mathbb{R}^{3}} \int_{\mathbb{R}^{3}}\left(\frac{u}{\sqrt{M}}-\left\langle\frac{u}{\sqrt{M}}\right\rangle\right) \frac{\varphi}{\sqrt{M}} \mathrm{~d} v \mathrm{~d} x=0 .
$$

The Poincaré inequality holds also true, with the same constant, since for any $u \in$ $\mathrm{D}\left(\mathcal{T}_{M}\right) \cap \operatorname{ker}\langle\cdot\rangle_{M}$ we can write

$$
\|u\|_{L^{2}\left(M^{-1}\right)}=\left\|\frac{u}{\sqrt{M}}\right\| \leq \frac{2 \pi}{\left|\omega_{c}\right|}\left\|\mathcal{T}\left(\frac{u}{\sqrt{M}}\right)\right\|=\frac{2 \pi}{\left|\omega_{c}\right|}\left\|\frac{\mathcal{T}_{M} u}{\sqrt{M}}\right\|=\frac{2 \pi}{\left|\omega_{c}\right|}\left\|\mathcal{T}_{M} u\right\|_{L^{2}\left(M^{-1}\right)} .
$$

From now on, for the sake of simplicity, we will use only the notations $\mathcal{T},\langle\cdot\rangle$, independently of acting on $L^{2}(\mathrm{~d} x \mathrm{~d} v)$ or $L^{2}\left(M^{-1} \mathrm{~d} x \mathrm{~d} v\right)$.

3. Average and first order differential operators. We intend to average transport operators, see (1.8). Moreover, in order to handle the Fokker-Planck kernel we will need to average second order differential operators. For doing that it is convenient to identify derivations which leave invariant $\operatorname{ker} \mathcal{T}$. It turns out that these derivations are those along the invariants

$$
\psi_{1}=x_{1}+\frac{v_{2}}{\omega_{c}}, \quad \psi_{2}=x_{2}-\frac{v_{1}}{\omega_{c}}, \quad \psi_{3}=x_{3}, \quad \psi_{4}=\sqrt{\left(v_{1}\right)^{2}+\left(v_{2}\right)^{2}}, \quad \psi_{5}=v_{3} .
$$


We introduce also $\psi_{0}=-\frac{\alpha}{\omega_{c}}$, with $\bar{v}=|\bar{v}| e^{i \alpha}, \alpha \in\left[0,2 \pi\left[\right.\right.$. Notice that $\psi_{0}$ has a jump of $\frac{2 \pi}{\omega_{c}}$ across $\bar{v} \in \mathbb{R}_{+} \times\{0\}$ but not its gradient with respect to $\bar{v}$

$$
\nabla_{\bar{v}} \alpha=-\frac{\perp \bar{v}}{|\bar{v}|^{2}}, \quad \nabla_{\bar{v}} \psi_{0}=\frac{\perp \bar{v}}{\omega_{c}|\bar{v}|^{2}}, \quad \mathcal{T} \psi_{0}=1 .
$$

The idea is to consider the fields $\left(b^{i}\right)_{0 \leq i \leq 5}$ such that

$$
b^{i} \cdot \nabla_{x, v} \psi_{j}=\delta_{j}^{i}, \quad 0 \leq i, j \leq 5 .
$$

Indeed, the map $(x, v) \rightarrow\left(\psi_{i}(x, v)\right)_{0 \leq i \leq 5}$ defines a change of coordinates

$$
\begin{gathered}
x_{1}=\psi_{1}+\frac{\psi_{4}}{\omega_{c}} \sin \left(\omega_{c} \psi_{0}\right), \quad x_{2}=\psi_{2}+\frac{\psi_{4}}{\omega_{c}} \cos \left(\omega_{c} \psi_{0}\right), \quad x_{3}=\psi_{3} \\
v_{1}=\psi_{4} \cos \left(\omega_{c} \psi_{0}\right), \quad v_{2}=-\psi_{4} \sin \left(\omega_{c} \psi_{0}\right), \quad v_{3}=\psi_{5} .
\end{gathered}
$$

Therefore any function $u=u(x, v)$ can be written $u(x, v)=U(\psi(x, v)), \psi=\left(\psi_{i}\right)_{0 \leq i \leq 5}$ and thus, for any $i \in\{0,1, \ldots, 5\}$ we have

$$
b^{i} \cdot \nabla_{x, v} u=b^{i} \cdot \sum_{j=0}^{5} \frac{\partial U}{\partial \psi_{j}}(\psi(x, v)) \nabla_{x, v} \psi_{j}=\frac{\partial U}{\partial \psi_{i}}(\psi(x, v)) .
$$

In other words the derivations $b^{i} \cdot \nabla_{x, v}$ act like $\partial_{\psi_{i}}, 0 \leq i \leq 5$. In particular if $u \in \operatorname{ker} \mathcal{T}$, meaning that $U$ does not depend on $\psi_{0}$, then $b^{i} \cdot \nabla_{x, v} u=\partial_{\psi_{i}} U(\psi(x, v))$ does not depend on $\psi_{0}$, saying that $\operatorname{ker} \mathcal{T}$ is left invariant by $b^{i} \cdot \nabla_{x, v}, 0 \leq i \leq 5$. The following result comes by direct computation and is left to the reader. For any smooth vector fields $\xi, \eta$ on $\mathbb{R}^{6}$, the notation $[\xi, \eta]$ stands for their Poisson bracket i.e.,

$$
[\xi, \eta]=\left(\xi \cdot \nabla_{x, v}\right) \eta-\left(\eta \cdot \nabla_{x, v}\right) \xi
$$

Proposition 3.1. The fields $\left(b^{i}\right)_{0 \leq i \leq 5}$ satisfying $b^{i} \cdot \nabla_{x, v} \psi_{j}=\delta_{j}^{i}, 0 \leq i, j \leq 5$ are given by

$$
\begin{gathered}
b^{0} \cdot \nabla_{x, v}=\bar{v} \cdot \nabla_{\bar{x}}+\omega_{c}{ }^{\perp} \bar{v} \cdot \nabla_{\bar{v}}, \quad b^{1} \cdot \nabla_{x, v}=\partial_{x_{1}}, \quad b^{2} \cdot \nabla_{x, v}=\partial_{x_{2}}, \quad b^{3} \cdot \nabla_{x, v}=\partial_{x_{3}} \\
b^{4} \cdot \nabla_{x, v}=-\frac{{ }^{\perp} \bar{v}}{\omega_{c}|\bar{v}|} \cdot \nabla_{\bar{x}}+\frac{\bar{v}}{|\bar{v}|} \cdot \nabla_{\bar{v}}, \quad b^{5} \cdot \nabla_{x, v}=\partial_{v_{3}} .
\end{gathered}
$$

Moreover the Poisson brackets between $\left(b^{i}\right)_{0 \leq i \leq 5}$ vanishes or equivalently the derivations $b^{i} \cdot \nabla_{x, v}, 0 \leq i \leq 5$ are commuting.

REMARK 3.1. Notice that $\left(b^{i}\right)_{i \neq 4}$ are divergence free and $\operatorname{div}_{x, v} b^{4}=\frac{1}{|\bar{v}|}$. 
We claim that the operators $u \rightarrow \operatorname{div}_{x, v}\left(u b^{i}\right)$, with domain

$$
\mathrm{D}\left(\operatorname{div}_{x, v}\left(\cdot b^{i}\right)\right)=\left\{u \in L^{2}\left(\mathbb{R}^{3} \times \mathbb{R}^{3}\right): \operatorname{div}_{x, v}\left(u b^{i}\right) \in L^{2}\left(\mathbb{R}^{3} \times \mathbb{R}^{3}\right)\right\}, \quad 0 \leq i \leq 5
$$

are commuting with the average operator. More generally we establish the following result.

Proposition 3.2. Assume that the field $c \cdot \nabla_{x, v}$ is in involution with $b \cdot \nabla_{x, v}=\bar{v} \cdot \nabla_{\bar{x}}+$ $\omega_{c}{ }^{\perp} \bar{v} \cdot \nabla_{\bar{v}}$ i.e., $[c, b]=0$. Then the operator $\operatorname{div}_{x, v}(\cdot c)$ is commuting with the average operator associated to the flow of $b \cdot \nabla_{x, v}$ that is, for any function $u \in \mathrm{D}\left(\operatorname{div}_{x, v}(\cdot c)\right)$ its average $\langle u\rangle$ belongs to $\mathrm{D}\left(\operatorname{div}_{x, v}(\cdot c)\right)$ and

$$
\operatorname{div}_{x, v}(\langle u\rangle c)=\left\langle\operatorname{div}_{x, v}(u c)\right\rangle
$$

Proof. Let us consider $u \in \mathrm{D}\left(\operatorname{div}_{x, v}(\cdot c)\right)$. For any $\varphi \in C_{c}^{1}\left(\mathbb{R}^{3} \times \mathbb{R}^{3}\right) \cap \operatorname{ker} \mathcal{T}$ we have

$$
\int_{\mathbb{R}^{3}} \int_{\mathbb{R}^{3}}\left\langle\operatorname{div}_{x, v}(u c)\right\rangle \varphi \mathrm{d} v \mathrm{~d} x=\int_{\mathbb{R}^{3}} \int_{\mathbb{R}^{3}} \operatorname{div}_{x, v}(u c) \varphi \mathrm{d} v \mathrm{~d} x=-\int_{\mathbb{R}^{3}} \int_{\mathbb{R}^{3}} u c \cdot \nabla_{x, v} \varphi \mathrm{d} v \mathrm{~d} x .
$$

But $\mathcal{T}\left(c \cdot \nabla_{x, v} \varphi\right)=c \cdot \nabla_{x, v}(\mathcal{T} \varphi)=0$ saying that $c \cdot \nabla_{x, v} \varphi \in \operatorname{ker} \mathcal{T}$ and thus

$$
\int_{\mathbb{R}^{3}} \int_{\mathbb{R}^{3}} u c \cdot \nabla_{x, v} \varphi \mathrm{d} v \mathrm{~d} x=\int_{\mathbb{R}^{3}} \int_{\mathbb{R}^{3}}\langle u\rangle c \cdot \nabla_{x, v} \varphi \mathrm{d} v \mathrm{~d} x .
$$

Combining (3.1), (3.2) we obtain for any $\varphi \in C_{c}^{1}\left(\mathbb{R}^{3} \times \mathbb{R}^{3}\right) \cap \operatorname{ker} \mathcal{T}$

$$
\int_{\mathbb{R}^{3}} \int_{\mathbb{R}^{3}}\left\langle\operatorname{div}_{x, v}(u c)\right\rangle \varphi \mathrm{d} v \mathrm{~d} x=-\int_{\mathbb{R}^{3}} \int_{\mathbb{R}^{3}}\langle u\rangle c \cdot \nabla_{x, v} \varphi \mathrm{d} v \mathrm{~d} x .
$$

Actually the previous equality holds also true for smooth functions $\varphi \in \operatorname{ker}\langle\cdot\rangle$. Indeed, by Proposition 2.2, for any smooth function $\varphi \in \operatorname{ker}\langle\cdot\rangle$ there is $\psi \in \mathrm{D}(\mathcal{T}) \cap \operatorname{ker}\langle\cdot\rangle$ such that $\mathcal{T} \psi=\varphi$ and thus $c \cdot \nabla_{x, v} \varphi=c \cdot \nabla_{x, v}(\mathcal{T} \psi)=\mathcal{T}\left(c \cdot \nabla_{x, v} \psi\right) \in$ Range $\mathcal{T}=\operatorname{ker}\langle\cdot\rangle$. Using now the orthogonality between $\operatorname{ker} \mathcal{T}$ and $\operatorname{ker}\langle\cdot\rangle$ we deduce that $\int_{\mathbb{R}^{3}} \int_{\mathbb{R}^{3}}\left\langle\operatorname{div}_{x, v}(u c)\right\rangle \varphi \mathrm{d} v \mathrm{~d} x=0=-\int_{\mathbb{R}^{3}} \int_{\mathbb{R}^{3}}\langle u\rangle c \cdot \nabla_{x, v} \varphi \mathrm{d} v \mathrm{~d} x, \quad \varphi \in C_{c}^{1}\left(\mathbb{R}^{3} \times \mathbb{R}^{3}\right) \cap \operatorname{ker}\langle\cdot\rangle$. Finally (3.3) is verified for any smooth $\varphi$, implying that

$$
\langle u\rangle \in \mathrm{D}\left(\operatorname{div}_{x, v}(\cdot c)\right) \text { and } \operatorname{div}_{x, v}(\langle u\rangle c)=\left\langle\operatorname{div}_{x, v}(u c)\right\rangle .
$$


We want to average transport operators, which are written in conservative forms. In order to obtain averaged model still written in conservative form, it is worth to establish the following commutation formula between average and divergence. For the sake of simplicity we discard all difficulties related to the required minimal smoothness.

Proposition 3.3. For any smooth field $\xi=\left(\xi_{x}, \xi_{v}\right) \in \mathbb{R}^{6}$ we have the equality

$$
\begin{aligned}
\left\langle\operatorname{div}_{x, v} \xi\right\rangle & =\operatorname{div}_{\bar{x}}\left\{\left\langle\xi_{\bar{x}}+\frac{{ }^{\perp} \xi_{\bar{v}}}{\omega_{c}}\right\rangle+\left\langle\xi_{\bar{v}} \cdot \frac{{ }^{\perp} \bar{v}}{|\bar{v}|}\right\rangle \frac{\bar{v}}{\omega_{c}|\bar{v}|}-\left\langle\xi_{\bar{v}} \cdot \frac{\bar{v}}{|\bar{v}|}\right\rangle \frac{{ }^{\perp} \bar{v}}{\omega_{c}|\bar{v}|}\right\}+\partial_{x_{3}}\left\langle\xi_{x_{3}}\right\rangle \\
& +\operatorname{div}_{\bar{v}}\left\{\left\langle\xi_{\bar{v}} \cdot \frac{{ }^{\perp} \bar{v}}{|\bar{v}|}\right\rangle \frac{{ }^{\perp} \bar{v}}{|\bar{v}|}+\left\langle\xi_{\bar{v}} \cdot \frac{\bar{v}}{|\bar{v}|}\right\rangle \frac{\bar{v}}{|\bar{v}|}\right\}+\partial_{v_{3}}\left\langle\xi_{v_{3}}\right\rangle .
\end{aligned}
$$

In particular we have for any smooth field $\xi_{x} \in \mathbb{R}^{3}$

$$
\left\langle\operatorname{div}_{x} \xi_{x}\right\rangle=\operatorname{div}_{x}\left\langle\xi_{x}\right\rangle
$$

and for any smooth field $\xi_{v} \in \mathbb{R}^{3}$

$$
\begin{aligned}
\left\langle\operatorname{div}_{v} \xi_{v}\right\rangle & =\operatorname{div}_{\bar{x}}\left\{\left\langle\frac{{ }^{\perp} \xi_{\bar{v}}}{\omega_{c}}\right\rangle+\left\langle\xi_{\bar{v}} \cdot \frac{\perp \bar{v}}{|\bar{v}|}\right\rangle \frac{\bar{v}}{\omega_{c}|\bar{v}|}-\left\langle\xi_{\bar{v}} \cdot \frac{\bar{v}}{|\bar{v}|}\right\rangle \frac{{ }^{\perp} \bar{v}}{\omega_{c}|\bar{v}|}\right\} \\
& +\operatorname{div}_{\bar{v}}\left\{\left\langle\xi_{\bar{v}} \cdot \frac{{ }_{\bar{v}}}{|\bar{v}|}\right\rangle \frac{{ }^{\perp} \bar{v}}{|\bar{v}|}+\left\langle\xi_{\bar{v}} \cdot \frac{\bar{v}}{|\bar{v}|}\right\rangle \frac{\bar{v}}{|\bar{v}|}\right\}+\partial_{v_{3}}\left\langle\xi_{v_{3}}\right\rangle
\end{aligned}
$$

Proof. By construction we have $\sum_{i=0}^{5} b^{i} \otimes \nabla_{x, v} \psi_{i}=I$ and thus

$$
\xi=\sum_{i=0}^{5}\left(\xi \cdot \nabla_{x, v} \psi_{i}\right) b^{i}
$$

The main statement follows thanks to Proposition 3.2, since we have

$$
\left\langle\operatorname{div}_{x, v} \xi\right\rangle=\left\langle\sum_{i=0}^{5} \operatorname{div}_{x, v}\left\{\left(\xi \cdot \nabla_{x, v} \psi_{i}\right) b^{i}\right\}\right\rangle=\operatorname{div}_{x, v}\left\{\sum_{i=0}^{5}\left\langle\xi \cdot \nabla_{x, v} \psi_{i}\right\rangle b^{i}\right\} .
$$

The other statements come by considering the fields $\left(\xi_{x}, 0\right)$ and $\left(0, \xi_{v}\right)$.

A direct consequence of Proposition 3.3 is the computation of the average for the transport operator in (1.6).

Proposition 3.4. Assume that the electric field derives from a smooth potential i.e., $E=-\nabla_{x} \phi$. Then for any $f \in C_{c}^{1}\left(\mathbb{R}^{3} \times \mathbb{R}^{3}\right) \cap \operatorname{ker} \mathcal{T}$ we have

$$
\left\langle\partial_{t} f+v_{3} \partial_{x_{3}} f+\frac{q}{m} E \cdot \nabla_{v} f+\mathcal{T} f^{1}\right\rangle=\partial_{t} f+\frac{\langle\perp \bar{E}\rangle}{B} \cdot \nabla_{\bar{x}} f+v_{3} \partial_{x_{3}} f+\frac{q}{m}\left\langle E_{3}\right\rangle \partial_{v_{3}} f .
$$


Proof. We can write

$$
\left\langle\partial_{t} f+v_{3} \partial_{x_{3}} f+\frac{q}{m} E \cdot \nabla_{v} f+\mathcal{T} f^{1}\right\rangle=\partial_{t} f+\left\langle v_{3} \partial_{x_{3}} f\right\rangle+\frac{q}{m}\left\langle E \cdot \nabla_{v} f\right\rangle
$$

since $\left\langle\mathcal{T} f^{1}\right\rangle=0$ and $\left\langle\partial_{t} f\right\rangle=\partial_{t}\langle f\rangle=\partial_{t} f$. The average of $v_{3} \partial_{x_{3}} f$ comes easily thanks to Proposition 3.2

$$
\left\langle v_{3} \partial_{x_{3}} f\right\rangle=\left\langle\operatorname{div}_{x, v}\left\{f v_{3} b^{3}\right\}\right\rangle=\operatorname{div}_{x, v}\left\{\left\langle f v_{3}\right\rangle b^{3}\right\}=\operatorname{div}_{x, v}\left\{f v_{3} b^{3}\right\}=v_{3} \partial_{x_{3}} f .
$$

Observe that $\mathcal{T}(f \phi)=f \bar{v} \cdot \nabla_{\bar{x}} \phi=-f \bar{v} \cdot \bar{E}$ and thus $\langle f \bar{v} \cdot \bar{E}\rangle=0$. Thanks to Proposition 3.3 one gets

$$
\begin{aligned}
\left\langle\operatorname{div}_{v}\{f E\}\right\rangle & =\operatorname{div}_{\bar{x}}\left\langle f \frac{\perp \bar{E}}{\omega_{c}}\right\rangle+\mathcal{T}\left\langle f \frac{\perp \bar{v} \cdot \bar{E}}{\omega_{c}|\bar{v}|^{2}}\right\rangle+\partial_{v_{3}}\left\langle f E_{3}\right\rangle \\
& =\operatorname{div}_{\bar{x}}\left\{f\left\langle\frac{\perp \bar{E}}{\omega_{c}}\right\rangle\right\}+\partial_{v_{3}}\left\{f\left\langle E_{3}\right\rangle\right\}
\end{aligned}
$$

implying that

$$
\frac{q}{m}\left\langle\operatorname{div}_{v}\{f E\}\right\rangle=\operatorname{div}_{\bar{x}}\left\{f \frac{\langle\perp \bar{E}\rangle}{B}\right\}+\frac{q}{m} \partial_{v_{3}}\left\{f\left\langle E_{3}\right\rangle\right\} .
$$

Using again Proposition 3.2 notice that

$$
\partial_{v_{3}}\left\langle E_{3}\right\rangle=\operatorname{div}_{x, v}\left\{\left\langle E_{3}\right\rangle b^{5}\right\}=\left\langle\operatorname{div}_{x, v}\left\{E_{3} b^{5}\right\}\right\rangle=\left\langle\partial_{v_{3}} E_{3}\right\rangle=0
$$

and

$$
\operatorname{div}_{\bar{x}}\langle\perp \bar{E}\rangle=\left\langle\operatorname{div}_{\bar{x}}{ }^{\perp} \bar{E}\right\rangle=0
$$

and our statement follows.

REMARK 3.2. We have proved that averaging the transport operator $a \cdot \nabla_{x, v}:=$ $v_{3} \partial_{x_{3}}+\frac{q}{m} E \cdot \nabla_{v}$ leads to $A \cdot \nabla_{x, v}:=\frac{\left\langle{ }^{\perp} \bar{E}\right\rangle}{B} \cdot \nabla_{\bar{x}}+v_{3} \partial_{x_{3}}+\frac{q}{m}\left\langle E_{3}\right\rangle \partial_{v_{3}}$ which verifies

$$
\left\langle a \cdot \nabla_{x, v} f\right\rangle=A \cdot \nabla_{x, v} f, \quad f \in C_{c}^{1}\left(\mathbb{R}^{3} \times \mathbb{R}^{3}\right) \cap \operatorname{ker} \mathcal{T} .
$$

By construction, the operator $A \cdot \nabla_{x, v}$ leaves invariant the subspace of smooth functions of $\operatorname{ker} \mathcal{T}$. By antisymmetry ( $\operatorname{since}^{\left.\operatorname{div}_{x, v} A=0\right)}$ it is easily seen that $A \cdot \nabla_{x, v}$ also leaves invariant the subspace of smooth functions in $\operatorname{ker}\langle\cdot\rangle$. Indeed, consider $h$ a zero average 
smooth function and let us prove that $\left\langle A \cdot \nabla_{x, v} h\right\rangle=0$ For any smooth $f$ in ker $\mathcal{T}$ we have

$$
\int_{\mathbb{R}^{3}} \int_{\mathbb{R}^{3}} A \cdot \nabla_{x, v} h f \mathrm{~d} v \mathrm{~d} x=-\int_{\mathbb{R}^{3}} \int_{\mathbb{R}^{3}} h A \cdot \nabla_{x, v} f \mathrm{~d} v \mathrm{~d} x=0
$$

by the orthogonality between $\operatorname{ker}\langle\cdot\rangle$ and $\operatorname{ker} \mathcal{T}$, and thus $\left\langle A \cdot \nabla_{x, v} h\right\rangle=0$. Finally $A \cdot \nabla_{x, v}$ is commuting with the average operator $\left\langle A \cdot \nabla_{x, v} f\right\rangle=A \cdot \nabla_{x, v}\langle f\rangle$ for any smooth $f$.

4. The relaxation collision operator. In this section we analyze the linear Boltzmann collision kernel $[18,17]$

$$
Q_{B}(f)(x, v)=\frac{1}{\tau} \int_{\mathbb{R}^{3}} s\left(v, v^{\prime}\right)\left\{M(v) f\left(x, v^{\prime}\right)-M\left(v^{\prime}\right) f(x, v)\right\} \mathrm{d} v^{\prime}
$$

where the scattering cross section satisfies

$$
s\left(v, v^{\prime}\right)=s\left(v^{\prime}, v\right), \quad 0<s_{0} \leq s\left(v, v^{\prime}\right) \leq S_{0}<+\infty, \quad v, v^{\prime} \in \mathbb{R}^{3} .
$$

We recall the standard properties of this operator. Here $Q_{B}^{ \pm}$denote the gain/loss relaxation collision operators

$$
Q_{B}^{+}(f)(v)=\frac{1}{\tau} \int_{\mathbb{R}^{3}} s\left(v, v^{\prime}\right) M(v) f\left(v^{\prime}\right) \mathrm{d} v^{\prime}, \quad Q_{B}^{-}(f)(v)=\frac{1}{\tau} \int_{\mathbb{R}^{3}} s\left(v, v^{\prime}\right) M\left(v^{\prime}\right) f(v) \mathrm{d} v^{\prime} .
$$

Proposition 4.1. Assume that the scattering cross section satisfies (4.2). Then

1. The gain/loss collision operators $Q_{B}^{ \pm}$are linear bounded operators on $L^{2}\left(M^{-1} \mathrm{~d} v\right)$, with $\left\|Q_{B}^{ \pm}\right\| \leq S_{0} / \tau$, and symmetric with respect to the scalar product of $L^{2}\left(M^{-1} \mathrm{~d} v\right)$.

2. For any $f \in L^{2}\left(M^{-1} \mathrm{~d} v\right)$ we have

$$
\int_{\mathbb{R}^{3}} Q_{B}(f)(v) f(v) \frac{\mathrm{d} v}{M}=-\frac{1}{2 \tau} \int_{\mathbb{R}^{3}} \int_{\mathbb{R}^{3}} s\left(v, v^{\prime}\right) M(v) M\left(v^{\prime}\right)\left[\frac{f(v)}{M(v)}-\frac{f\left(v^{\prime}\right)}{M\left(v^{\prime}\right)}\right]^{2} \mathrm{~d} v^{\prime} \mathrm{d} v \leq 0 .
$$

We want to average $Q_{B}(f)$ for functions $f$ satisfying the constraint (1.5). In this section the operators $\mathcal{T}$ and $\langle\cdot\rangle$ should be understood in the $L^{2}\left(M^{-1} \mathrm{~d} x \mathrm{~d} v\right)$ framework. We need to compute the average of functions like $\int_{\mathbb{R}^{3}} C\left(v, v^{\prime}\right) f\left(x, v^{\prime}\right) \mathrm{d} v^{\prime}$ where $C\left(v, v^{\prime}\right)$ is a given function. The corresponding result in the bidimensional framework has been announced in [5]. We will see that we only need to consider functions $C$ which are 
left invariant by any rotation around $e_{3}=(0,0,1)$. Therefore we assume that for any orthogonal matrix $\mathcal{O} \in \mathcal{M}_{3}(\mathbb{R})$ such that $\mathcal{O} e_{3}=e_{3}$ we have

$$
C\left({ }^{t} \mathcal{O} v,{ }^{t} \mathcal{O} v^{\prime}\right)=C\left(v, v^{\prime}\right), \quad v, v^{\prime} \in \mathbb{R}^{3} .
$$

These functions are precisely those depending only on $|\bar{v}|, v_{3},\left|\overline{v^{\prime}}\right|, v_{3}^{\prime}$ and the angle between $\bar{v}$ and $\overline{v^{\prime}}$

$$
C\left(v, v^{\prime}\right)=\tilde{C}\left(|\bar{v}|, v_{3},\left|\overline{v^{\prime}}\right|, v_{3}^{\prime}, \varphi\right), \quad \varphi=\arg \overline{v^{\prime}}-\arg \bar{v} .
$$

Proposition 4.2. Assume that the function $C\left(v, v^{\prime}\right)$ satisfies (4.3) and belongs to the space $L^{2}\left(M^{-1}(v) M\left(v^{\prime}\right) \mathrm{d} v \mathrm{~d} v^{\prime}\right)$. Then for any function $f \in \operatorname{ker} \mathcal{T}$ we have

$$
\left\langle\int_{\mathbb{R}^{3}} C\left(v, v^{\prime}\right) f\left(x, v^{\prime}\right) \mathrm{d} v^{\prime}\right\rangle(x, v)=\omega_{c}^{2} \int_{\mathbb{R}^{2}} \int_{\mathbb{R}^{3}} \mathcal{C}\left(|\bar{v}|, v_{3},\left|\overline{v^{\prime}}\right|, v_{3}^{\prime}, z\right) f\left(\overline{x^{\prime}}, x_{3}, v^{\prime}\right) \mathrm{d} v^{\prime} \mathrm{d} x_{1}^{\prime} \mathrm{d} x_{2}^{\prime}
$$

where $z=\omega_{c} \bar{x}+{ }^{\perp} \bar{v}-\left(\omega_{c} \overline{x^{\prime}}+{ }^{\perp} \overline{v^{\prime}}\right)$

$$
\mathcal{C}\left(r, v_{3}, r^{\prime}, v_{3}^{\prime}, z\right)=\frac{\tilde{C}\left(r, v_{3}, r^{\prime}, v_{3}^{\prime}, \varphi\right)+\tilde{C}\left(r, v_{3}, r^{\prime}, v_{3}^{\prime},-\varphi\right)}{2 \pi^{2} \sqrt{|z|^{2}-\left(r-r^{\prime}\right)^{2}} \sqrt{\left(r+r^{\prime}\right)^{2}-|z|^{2}}} \mathbf{1}_{\left\{\left|r-r^{\prime}\right|<|z|<r+r^{\prime}\right\}}
$$

and for any $|z| \in\left(\left|r-r^{\prime}\right|, r+r^{\prime}\right), \varphi \in(0, \pi)$ is the unique angle such that

$$
|z|^{2}=r^{2}+\left(r^{\prime}\right)^{2}-2 r r^{\prime} \cos \varphi
$$

Proof. For $(x, v) \in \mathbb{R}^{3} \times \mathbb{R}^{3}$ we have

$$
\left(\int_{\mathbb{R}^{3}} C\left(v, v^{\prime}\right) f\left(x, v^{\prime}\right) \mathrm{d} v^{\prime}\right)^{2} \leq \int_{\mathbb{R}^{3}}\left(C\left(v, v^{\prime}\right)\right)^{2} M\left(v^{\prime}\right) \mathrm{d} v^{\prime} \int_{\mathbb{R}^{3}} \frac{\left(f\left(x, v^{\prime}\right)\right)^{2}}{M\left(v^{\prime}\right)} \mathrm{d} v^{\prime}
$$

implying that

$$
\left\|\int_{\mathbb{R}^{3}} C\left(v, v^{\prime}\right) f\left(x, v^{\prime}\right) \mathrm{d} v^{\prime}\right\|_{L^{2}\left(M^{-1} \mathrm{~d} x \mathrm{~d} v\right)} \leq\|C\|_{L^{2}\left(M^{-1}(v) M\left(v^{\prime}\right) \mathrm{d} v \mathrm{~d} v^{\prime}\right)}\|f\|_{L^{2}\left(M^{-1} \mathrm{~d} x \mathrm{~d} v\right)} .
$$

Therefore the function $(x, v) \rightarrow \int_{\mathbb{R}^{3}} C\left(v, v^{\prime}\right) f\left(x, v^{\prime}\right) \mathrm{d} v^{\prime}$ belongs to $L^{2}\left(M^{-1} \mathrm{~d} x \mathrm{~d} v\right)$ and can be averaged in $L^{2}\left(M^{-1} \mathrm{~d} x \mathrm{~d} v\right)$. Consider $(x, v) \in \mathbb{R}^{3} \times \mathbb{R}^{3}$. By formula (2.1) we have

$$
\begin{aligned}
I: & =\left\langle\int_{\mathbb{R}^{3}} C\left(v, v^{\prime}\right) f\left(x, v^{\prime}\right) \mathrm{d} v^{\prime}\right\rangle(x, v) \\
& =\frac{1}{2 \pi} \int_{0}^{2 \pi} \int_{\mathbb{R}^{3}} C\left(|\bar{v}| e^{i \alpha}, v_{3}, v^{\prime}\right) f\left(\bar{x}+\frac{\perp_{\bar{v}}}{\omega_{c}}-\frac{\perp\left\{|\bar{v}| e^{i \alpha}\right\}}{\omega_{c}}, x_{3}, v^{\prime}\right) \mathrm{d} v^{\prime} \mathrm{d} \alpha .
\end{aligned}
$$


For any fixed $\alpha \in[0,2 \pi)$ we use the cylindrical coordinates

$$
v^{\prime}=\left(r^{\prime} e^{i(\varphi+\alpha)}, v_{3}^{\prime}\right), \quad r^{\prime} \in \mathbb{R}_{+}, \quad \varphi \in[-\pi, \pi)
$$

and therefore

$$
\begin{aligned}
I & =\frac{1}{2 \pi} \int_{0}^{2 \pi} \int_{\mathbb{R}} \int_{-\pi}^{\pi} \int_{\mathbb{R}_{+}} C\left(|\bar{v}| e^{i \alpha}, v_{3}, r^{\prime} e^{i(\varphi+\alpha)}, v_{3}^{\prime}\right) \\
& \times f\left(\bar{x}+\frac{{ }^{\perp} \bar{v}}{\omega_{c}}-\frac{\perp\left\{|\bar{v}| e^{i \alpha}\right\}}{\omega_{c}}, x_{3}, r^{\prime} e^{i(\varphi+\alpha)}, v_{3}^{\prime}\right) r^{\prime} \mathrm{d} r^{\prime} \mathrm{d} \varphi \mathrm{d} v_{3}^{\prime} \mathrm{d} \alpha .
\end{aligned}
$$

But $f \in \operatorname{ker} \mathcal{T}$ and thus there is $g$ such that

$$
f(x, v)=g\left(\bar{x}+\frac{{ }^{\perp} \bar{v}}{\omega_{c}}, x_{3},|\bar{v}|, v_{3}\right)
$$

implying that

$f\left(\bar{x}+\frac{{ }^{\perp} \bar{v}}{\omega_{c}}-\frac{{ }^{\perp}\left\{|\bar{v}| e^{i \alpha}\right\}}{\omega_{c}}, x_{3}, r^{\prime} e^{i(\varphi+\alpha)}, v_{3}^{\prime}\right)=g\left(\bar{x}+\frac{{ }^{\perp} \bar{v}}{\omega_{c}}-\frac{{ }^{\perp}\left\{|\bar{v}| e^{i \alpha}\right\}}{\omega_{c}}+\frac{{ }^{\perp}\left\{r^{\prime} e^{i(\varphi+\alpha)}\right\}}{\omega_{c}}, x_{3}, r^{\prime}, v_{3}^{\prime}\right)$.

By one hand notice that $r^{\prime} e^{i(\varphi+\alpha)}-|\bar{v}| e^{i \alpha}=l e^{i(\psi+\alpha)}$ where $l^{2}=r^{2}+\left(r^{\prime}\right)^{2}-2 r r^{\prime} \cos \varphi$, $r=|\bar{v}|$ and $\psi$ depends on $r, r^{\prime}, \varphi$ but not on $\alpha$. By the other hand, since $C$ is invariant by rotation around $e_{3}$ we deduce that

$$
C\left(r e^{i \alpha}, v_{3}, r^{\prime} e^{i(\varphi+\alpha)}, v_{3}^{\prime}\right)=\tilde{C}\left(r, v_{3}, r^{\prime}, v_{3}^{\prime}, \varphi\right), \quad \varphi=\arg \overline{v^{\prime}}-\arg \bar{v}
$$

The map $\varphi \rightarrow l(\varphi)=\sqrt{r^{2}+\left(r^{\prime}\right)^{2}-2 r r^{\prime} \cos \varphi}$ defines a coordinate change between $\varphi \in(0, \pi)$ and $l \in\left(\left|r-r^{\prime}\right|, r+r^{\prime}\right)$ and

$$
\mathrm{d} \varphi=\frac{2 l \mathrm{~d} l}{\sqrt{l^{2}-\left(r-r^{\prime}\right)^{2}} \sqrt{\left(r+r^{\prime}\right)^{2}-l^{2}}} .
$$


By Fubini theorem one gets

$$
\begin{aligned}
I & =\frac{1}{2 \pi} \int_{\mathbb{R}} \int_{-\pi}^{\pi} \int_{\mathbb{R}_{+}} \int_{0}^{2 \pi} \tilde{C}\left(r, v_{3}, r^{\prime}, v_{3}^{\prime}, \varphi\right) g\left(\bar{x}+\frac{{ }^{\prime} \bar{v}}{\omega_{c}}+\frac{\perp\left\{l e^{i(\psi+\alpha)}\right\}}{\omega_{c}}, x_{3}, r^{\prime}, v_{3}^{\prime}\right) r^{\prime} \mathrm{d} \alpha \mathrm{d} r^{\prime} \mathrm{d} \varphi \mathrm{d} v_{3}^{\prime} \\
& =\frac{1}{2 \pi} \int_{\mathbb{R}} \int_{-\pi}^{\pi} \int_{\mathbb{R}_{+}} \int_{0}^{2 \pi} \tilde{C}\left(r, v_{3}, r^{\prime}, v_{3}^{\prime}, \varphi\right) g\left(\bar{x}+\frac{\perp \bar{v}}{\omega_{c}}+\frac{\perp\left\{l e^{i \alpha}\right\}}{\omega_{c}}, x_{3}, r^{\prime}, v_{3}^{\prime}\right) r^{\prime} \mathrm{d} \alpha \mathrm{d} r^{\prime} \mathrm{d} \varphi \mathrm{d} v_{3}^{\prime} \\
& =\frac{1}{2 \pi} \int_{\mathbb{R}} \int_{\mathbb{R}_{+}} \int_{0}^{2 \pi} \int_{0}^{\pi}\left\{\tilde{C}\left(r, v_{3}, r^{\prime}, v_{3}^{\prime}, \varphi\right)+\tilde{C}\left(r, v_{3}, r^{\prime}, v_{3}^{\prime},-\varphi\right)\right\} \\
& \times g\left(\bar{x}+\frac{{ }^{\prime} \bar{v}}{\omega_{c}}+\frac{\perp\left\{(\varphi) e^{i \alpha}\right\}}{\omega_{c}}, x_{3}, r^{\prime}, v_{3}^{\prime}\right) r^{\prime} \mathrm{d} \varphi \mathrm{d} \alpha \mathrm{d} r^{\prime} \mathrm{d} v_{3}^{\prime} \\
& =\frac{1}{2 \pi} \int_{\mathbb{R}} \int_{\mathbb{R}_{+}} \int_{0}^{2 \pi} \int_{\left|r-r^{\prime}\right|}^{r+r^{\prime}}\left\{\tilde{C}\left(r, v_{3}, r^{\prime}, v_{3}^{\prime}, \varphi(l)\right)+\tilde{C}\left(r, v_{3}, r^{\prime}, v_{3}^{\prime},-\varphi(l)\right)\right\} \\
& \times g\left(\bar{x}+\frac{{ }^{\prime} \bar{v}}{\omega_{c}}+\frac{\perp\left\{l e^{i \alpha}\right\}}{\omega_{c}}, x_{3}, r^{\prime}, v_{3}^{\prime}\right) \frac{2 l r^{\prime} \mathrm{d} l \mathrm{~d} \alpha \mathrm{d} r^{\prime} \mathrm{d} v_{3}^{\prime}}{\sqrt{l^{2}-\left(r-r^{\prime}\right)^{2}} \sqrt{\left(r+r^{\prime}\right)^{2}-l^{2}}} .
\end{aligned}
$$

For any $\alpha^{\prime} \in[0,2 \pi)$ we have

$$
g\left(\bar{x}+\frac{{ }^{\perp} \bar{v}}{\omega_{c}}+\frac{{ }^{\perp}\left\{l e^{i \alpha}\right\}}{\omega_{c}}, x_{3}, r^{\prime}, v_{3}^{\prime}\right)=f\left(\bar{x}+\frac{{ }^{\perp} \bar{v}}{\omega_{c}}+\frac{{ }^{\perp}\left\{l e^{i \alpha}\right\}}{\omega_{c}}-\frac{{ }^{\perp}\left\{r^{\prime} e^{i \alpha^{\prime}}\right\}}{\omega_{c}}, x_{3}, r^{\prime} e^{i \alpha^{\prime}}, v_{3}^{\prime}\right)
$$

and performing the change of coordinates $v^{\prime}=\left(r^{\prime} e^{i \alpha^{\prime}}, v_{3}^{\prime}\right)$ leads to

$$
\begin{aligned}
& I=\frac{1}{2 \pi^{2}} \int_{0}^{2 \pi} \int_{\mathbb{R}_{+}} \int_{\mathbb{R}} \int_{0}^{2 \pi} \int_{\mathbb{R}_{+}}\left\{\tilde{C}\left(r, v_{3}, r^{\prime}, v_{3}^{\prime}, \varphi(l)\right)+\tilde{C}\left(r, v_{3}, r^{\prime}, v_{3}^{\prime},-\varphi(l)\right)\right\} \\
& \times f\left(\bar{x}+\frac{{ }^{\perp} \bar{v}}{\omega_{c}}+\frac{{ }^{\perp}\left\{l e^{i \alpha}\right\}}{\omega_{c}}-\frac{{ }^{\perp}\left\{r^{\prime} e^{i \alpha^{\prime}}\right\}}{\omega_{c}}, x_{3}, r^{\prime} e^{i \alpha^{\prime}}, v_{3}^{\prime}\right) \frac{\mathbf{1}_{\left\{\left|r-r^{\prime}\right|<l<r+r^{\prime}\right\}} r^{\prime} \mathrm{d} r^{\prime} \mathrm{d} \alpha^{\prime} \mathrm{d} v_{3}^{\prime} l \mathrm{~d} l \mathrm{~d} \alpha}{\sqrt{l^{2}-\left(r-r^{\prime}\right)^{2}} \sqrt{\left(r+r^{\prime}\right)^{2}-l^{2}}} \\
& =\frac{1}{2 \pi^{2}} \int_{0}^{2 \pi} \int_{\mathbb{R}_{+}} \int_{\mathbb{R}^{3}}\left\{\tilde{C}\left(r, v_{3},\left|\overline{v^{\prime}}\right|, v_{3}^{\prime}, \varphi(l)\right)+\tilde{C}\left(r, v_{3},\left|\overline{v^{\prime}}\right|, v_{3}^{\prime},-\varphi(l)\right)\right\} \\
& \times f\left(\bar{x}+\frac{{ }^{\perp} \bar{v}}{\omega_{c}}+\frac{{ }^{\perp}\left\{l e^{i \alpha}\right\}}{\omega_{c}}-\frac{{ }^{\perp} \overline{v^{\prime}}}{\omega_{c}}, x_{3}, v^{\prime}\right) \frac{\mathbf{1}_{\left\{|| \bar{v}|-| \overline{v^{\prime}}||<l<|\bar{v}|+\left|\overline{v^{\prime}}\right|\right\}} \mathrm{d} v^{\prime} l \mathrm{~d} l \mathrm{~d} \alpha}{\sqrt{l^{2}-\left(|\bar{v}|-\left|\overline{v^{\prime}}\right|\right)^{2}} \sqrt{\left(|\bar{v}|+\left|\overline{v^{\prime}}\right|\right)^{2}-l^{2}}} .
\end{aligned}
$$

Using the notation

$$
\mathcal{C}\left(r, v_{3}, r^{\prime}, v_{3}^{\prime}, z\right)=\frac{\tilde{C}\left(r, v_{3}, r^{\prime}, v_{3}^{\prime}, \varphi\right)+\tilde{C}\left(r, v_{3}, r^{\prime}, v_{3}^{\prime},-\varphi\right)}{2 \pi^{2} \sqrt{|z|^{2}-\left(r-r^{\prime}\right)^{2}} \sqrt{\left(r+r^{\prime}\right)^{2}-|z|^{2}}} \mathbf{1}_{\left\{\left|r-r^{\prime}\right|<|z|<r+r^{\prime}\right\}}
$$

where for any $|z| \in\left(\left|r-r^{\prime}\right|, r+r^{\prime}\right), \varphi \in(0, \pi)$ is the unique angle such that

$$
|z|^{2}=r^{2}+\left(r^{\prime}\right)^{2}-2 r r^{\prime} \cos \varphi
$$


one gets

$I=\int_{\mathbb{R}^{3}} \int_{0}^{2 \pi} \int_{\mathbb{R}_{+}} \mathcal{C}\left(|\bar{v}|, v_{3},\left|\overline{v^{\prime}}\right|, v_{3}^{\prime},-^{\perp}\left\{l e^{i \alpha}\right\}\right) f\left(\bar{x}+\frac{{ }^{\perp} \bar{v}}{\omega_{c}}+\frac{\perp\left\{l e^{i \alpha}\right\}}{\omega_{c}}-\frac{\perp \overline{v^{\prime}}}{\omega_{c}}, x_{3}, v^{\prime}\right) l \mathrm{~d} l \mathrm{~d} \alpha \mathrm{d} v^{\prime}$.

We take as new coordinates

$$
\overline{x^{\prime}}=\bar{x}+\frac{\perp \bar{v}}{\omega_{c}}+\frac{\perp\left\{l e^{i \alpha}\right\}}{\omega_{c}}-\frac{\perp \overline{v^{\prime}}}{\omega_{c}}
$$

Observing that $\operatorname{det} \frac{\partial\left(x_{1}^{\prime}, x_{2}^{\prime}\right)}{\partial(l, \alpha)}=\frac{l}{\omega_{c}^{2}}$ we deduce that

$$
I=\omega_{c}^{2} \int_{\mathbb{R}^{2}} \int_{\mathbb{R}^{3}} \mathcal{C}\left(|\bar{v}|, v_{3},\left|\overline{v^{\prime}}\right|, v_{3}^{\prime},\left(\omega_{c} \bar{x}+{ }^{\perp} \bar{v}\right)-\left(\omega_{c} \overline{x^{\prime}}+{ }^{\perp} \overline{v^{\prime}}\right)\right) f\left(x_{1}^{\prime}, x_{2}^{\prime}, x_{3}, v^{\prime}\right) \mathrm{d} v^{\prime} \mathrm{d} x_{1}^{\prime} \mathrm{d} x_{2}^{\prime}
$$

REMARK 4.1. The constraint $\mathcal{T} f=0$ allows us to reduce the right hand side of (4.4) to a four dimensional integral. Indeed, thanks to (4.6) we obtain, using the notation

$$
\begin{aligned}
\bar{y} & =\bar{x}+\frac{\perp \bar{v}}{\omega_{c}} \\
I & =\omega_{c}^{2} \int_{\mathbb{R}^{2}} \int_{\mathbb{R}^{3}} \mathcal{C}\left(|\bar{v}|, v_{3},\left|\overline{v^{\prime}}\right|, v_{3}^{\prime},\left(\omega_{c} \bar{x}+{ }^{\perp} \bar{v}\right)-\left(\omega_{c} \overline{x^{\prime}}+{ }^{\perp} \overline{v^{\prime}}\right)\right) g\left(\overline{x^{\prime}}+\frac{\perp \overline{v^{\prime}}}{\omega_{c}}, x_{3},\left|\overline{v^{\prime}}\right|, v_{3}^{\prime}\right) \mathrm{d} v^{\prime} \mathrm{d} x_{1}^{\prime} \mathrm{d} x_{2}^{\prime} \\
& =\omega_{c}^{2} \int_{\mathbb{R}^{2}} \int_{\mathbb{R}^{3}} \mathcal{C}\left(|\bar{v}|, v_{3},\left|\overline{v^{\prime}}\right|, v_{3}^{\prime}, \omega_{c}\left(\bar{y}-\overline{y^{\prime}}\right)\right) g\left(\overline{y^{\prime}}, x_{3},\left|\overline{v^{\prime}}\right|, v_{3}^{\prime}\right) \mathrm{d} v^{\prime} \mathrm{d} y_{1}^{\prime} \mathrm{d} y_{2}^{\prime} \\
& =2 \pi \omega_{c}^{2} \int_{\mathbb{R}^{2}} \int_{\mathbb{R}} \int_{\mathbb{R}_{+}} \mathcal{C}\left(|\bar{v}|, v_{3}, r^{\prime}, v_{3}^{\prime}, \omega_{c}\left(\bar{y}-\overline{y^{\prime}}\right)\right) g\left(\overline{y^{\prime}}, x_{3}, r^{\prime}, v_{3}^{\prime}\right) r^{\prime} \mathrm{d} r^{\prime} \mathrm{d} v_{3}^{\prime} \mathrm{d} \overline{y^{\prime}} .
\end{aligned}
$$

We prefer to keep the five dimensional integral representation since, in the sequel, we will introduce similar integral terms, but with densities $f$ not satisfying the constraint $\mathcal{T} f=0$.

REMARK 4.2. If the function $\tilde{C}\left(r, v_{3}, r^{\prime}, v_{3}^{\prime}, \varphi\right)$ is odd with respect to $\varphi$, then $\mathcal{C}=0$ and

$$
\left\langle\int_{\mathbb{R}^{3}} C\left(v, v^{\prime}\right) f\left(x, v^{\prime}\right) \mathrm{d} v^{\prime}\right\rangle=0, \quad f \in \operatorname{ker} \mathcal{T}
$$

REMARK 4.3. Let $\chi$ be the function

$$
\chi\left(r, r^{\prime}, z\right)=\frac{\mathbf{1}_{\left\{\left|r-r^{\prime}\right|<|z|<r+r^{\prime}\right\}}}{\pi^{2} \sqrt{|z|^{2}-\left(r-r^{\prime}\right)^{2}} \sqrt{\left(r+r^{\prime}\right)^{2}-|z|^{2}}}
$$

for any $r, r^{\prime} \in \mathbb{R}_{+}, z \in \mathbb{R}^{2}$. Then for every $r, r^{\prime} \in \mathbb{R}_{+}, \chi\left(r, r^{\prime}, z\right) \mathrm{d} z$ is a probability measure on $\mathbb{R}^{2}$

$$
\int_{\mathbb{R}^{2}} \chi\left(r, r^{\prime}, z\right) \mathrm{d} z=1, \quad r, r^{\prime} \in \mathbb{R}_{+}
$$


and $\left\langle\int_{\mathbb{R}^{3}} C\left(v, v^{\prime}\right) f\left(x, v^{\prime}\right) \mathrm{d} v^{\prime}\right\rangle$ appears as a convolution with respect to the invariants $\omega_{c} \bar{x}+{ }^{\perp} \bar{v}$. Indeed, using the formula

$$
f\left(\overline{x^{\prime}}, x_{3}, v^{\prime}\right)=g\left(\overline{x^{\prime}}+\frac{\perp \overline{v^{\prime}}}{\omega_{c}}, x_{3},\left|\overline{v^{\prime}}\right|, v_{3}^{\prime}\right)
$$

we obtain

$$
\begin{aligned}
\left\langle\int_{\mathbb{R}^{3}} C\left(v, v^{\prime}\right) f\left(x, v^{\prime}\right) \mathrm{d} v^{\prime}\right\rangle(x, v) & =\int_{\mathbb{R}^{3}} \int_{\mathbb{R}^{2}} \mathcal{C}\left(|\bar{v}|, v_{3},\left|\overline{v^{\prime}}\right|, v_{3}^{\prime},\left(\omega_{c} \bar{x}+{ }^{\perp} \bar{v}\right)-\left(\omega_{c} \overline{x^{\prime}}+{ }^{\perp} \overline{v^{\prime}}\right)\right) \\
& \times g\left(\overline{x^{\prime}}+\frac{{ }^{\perp} \overline{v^{\prime}}}{\omega_{c}}, x_{3},\left|\overline{v^{\prime}}\right|, v_{3}^{\prime}\right) \mathrm{d}\left(\omega_{c} \overline{x^{\prime}}+{ }^{\perp} \overline{v^{\prime}}\right) \mathrm{d} v^{\prime}
\end{aligned}
$$

REMARK 4.4. The conclusion of Proposition 4.2 also holds true for bounded functions $f$ which are constant along the flow (1.7), provided that $C\left(v, v^{\prime}\right) \in L^{\infty}\left(\mathrm{d} v ; L^{1}\left(\mathrm{~d} v^{\prime}\right)\right)$ and satisfies (4.3). Indeed, in this case $f \rightarrow \int_{\mathbb{R}^{3}} C\left(v, v^{\prime}\right) f\left(x, v^{\prime}\right) \mathrm{d} v^{\prime}$ is bounded on $L^{\infty}(\mathrm{d} x \mathrm{~d} v)$

$$
\left\|\int_{\mathbb{R}^{3}} C\left(v, v^{\prime}\right) f\left(x, v^{\prime}\right) \mathrm{d} v^{\prime}\right\|_{L^{\infty}(\mathrm{d} x \mathrm{~d} v)} \leq\|C\|_{L^{\infty}\left(\mathrm{d} v ; L^{1}\left(\mathrm{~d} v^{\prime}\right)\right)}\|f\|_{L^{\infty}(\mathrm{d} x \mathrm{~d} v)}
$$

and using the $L^{\infty}$ version of the average operator, the same computations as those in the proof of Proposition 4.2 show that

$$
\left\langle\int_{\mathbb{R}^{3}} C\left(v, v^{\prime}\right) f\left(x, v^{\prime}\right) \mathrm{d} v^{\prime}\right\rangle(x, v)=\omega_{c}^{2} \int_{\mathbb{R}^{2}} \int_{\mathbb{R}^{3}} \mathcal{C}\left(|\bar{v}|, v_{3},\left|\overline{v^{\prime}}\right|, v_{3}^{\prime}, z\right) f\left(\overline{x^{\prime}}, x_{3}, v^{\prime}\right) \mathrm{d} v^{\prime} \mathrm{d} x_{1}^{\prime} \mathrm{d} x_{2}^{\prime} .
$$

Corollary 4.1. Assume that the scattering cross section satisfies (4.2) and

$$
s\left(v, v^{\prime}\right)=\sigma\left(\left|v-v^{\prime}\right|\right), \quad v, v^{\prime} \in \mathbb{R}^{3}
$$

for some function $\sigma: \mathbb{R}_{+} \rightarrow \mathbb{R}_{+}$. Then for any $f \in \operatorname{ker} \mathcal{T}$ we have

$\left\langle Q_{B} f\right\rangle(x, v)=\frac{\omega_{c}^{2}}{\tau} \int_{\mathbb{R}^{2}} \int_{\mathbb{R}^{3}} \mathcal{S}\left(|\bar{v}|, v_{3},\left|\overline{v^{\prime}}\right|, v_{3}^{\prime}, z\right)\left\{M(v) f\left(\overline{x^{\prime}}, x_{3}, v^{\prime}\right)-M\left(v^{\prime}\right) f(x, v)\right\} \mathrm{d} v^{\prime} \mathrm{d} x_{1}^{\prime} \mathrm{d} x_{2}^{\prime}$ with $z=\omega_{c} \bar{x}+{ }^{\perp} \bar{v}-\left(\omega_{c} \overline{x^{\prime}}+{ }^{\perp} \overline{v^{\prime}}\right)$ and

$$
\mathcal{S}\left(r, v_{3}, r^{\prime}, v_{3}^{\prime}, z\right)=\sigma\left(\sqrt{|z|^{2}+\left(v_{3}-v_{3}^{\prime}\right)^{2}}\right) \chi\left(r, r^{\prime}, z\right)
$$

Proof. Clearly the function $C\left(v, v^{\prime}\right)=\sigma\left(\left|v-v^{\prime}\right|\right) M(v)$ satisfies (4.3), belongs to $L^{2}\left(M^{-1}(v) M\left(v^{\prime}\right) \mathrm{d} v \mathrm{~d} v^{\prime}\right)$ and we have

$$
\begin{gathered}
\tilde{s}\left(r, v_{3}, r^{\prime}, v_{3}^{\prime}, \varphi\right)=\sigma\left(\sqrt{r^{2}+\left(r^{\prime}\right)^{2}-2 r r^{\prime} \cos \varphi+\left(v_{3}-v_{3}^{\prime}\right)^{2}}\right) \\
\mathcal{S}\left(r, v_{3}, r^{\prime}, v_{3}^{\prime}, z\right)=\sigma\left(\sqrt{|z|^{2}+\left(v_{3}-v_{3}^{\prime}\right)^{2}}\right) \chi\left(r, r^{\prime}, z\right) .
\end{gathered}
$$


Thanks to Proposition 4.2 we obtain, with $z=\left(\omega_{c} \bar{x}+{ }^{\perp} \bar{v}\right)-\left(\omega_{c} \overline{x^{\prime}}+{ }^{\perp} \overline{v^{\prime}}\right)$

$$
\left\langle\int_{\mathbb{R}^{3}} s\left(v, v^{\prime}\right) M(v) f\left(x, v^{\prime}\right) \mathrm{d} v^{\prime}\right\rangle=\omega_{c}^{2} \int_{\mathbb{R}^{2}} \int_{\mathbb{R}^{3}} \mathcal{S}\left(|\bar{v}|, v_{3},\left|\overline{v^{\prime}}\right|, v_{3}^{\prime}, z\right) M(v) f\left(\overline{x^{\prime}}, x_{3}, v^{\prime}\right) \mathrm{d} v^{\prime} \mathrm{d} x_{1}^{\prime} \mathrm{d} x_{2}^{\prime} .
$$

Since $f$ belongs to $L^{2}\left(M^{-1} \mathrm{~d} x \mathrm{~d} v\right)$ and remains constant along the flow (1.7) we have

$$
\left\langle\int_{\mathbb{R}^{3}} s\left(v, v^{\prime}\right) M\left(v^{\prime}\right) f(x, v) \mathrm{d} v^{\prime}\right\rangle=f(x, v)\left\langle\int_{\mathbb{R}^{3}} s\left(v, v^{\prime}\right) M\left(v^{\prime}\right) \mathrm{d} v^{\prime}\right\rangle
$$

where the first average operator should be understood in the $L^{2}\left(M^{-1} \mathrm{~d} x \mathrm{~d} v\right)$ setting and the second one in the $L^{\infty}(\mathrm{d} x \mathrm{~d} v)$ setting. Remark 4.4 applied with $C\left(v, v^{\prime}\right)=$ $s\left(v, v^{\prime}\right) M\left(v^{\prime}\right) \in L^{\infty}\left(\mathrm{d} v ; L^{1}\left(\mathrm{~d} v^{\prime}\right)\right)$ and the constant function $1 \in L^{\infty}(\mathrm{d} x \mathrm{~d} v)$ yields

$$
\left\langle\int_{\mathbb{R}^{3}} s\left(v, v^{\prime}\right) M\left(v^{\prime}\right) \mathrm{d} v^{\prime}\right\rangle=\omega_{c}^{2} \int_{\mathbb{R}^{2}} \int_{\mathbb{R}^{3}} \mathcal{S}\left(|\bar{v}|, v_{3},\left|\overline{v^{\prime}}\right|, v_{3}^{\prime}, z\right) M\left(v^{\prime}\right) \mathrm{d} v^{\prime} \mathrm{d} x_{1}^{\prime} \mathrm{d} x_{2}^{\prime} .
$$

Therefore we obtain

$$
\left\langle\int_{\mathbb{R}^{3}} s\left(v, v^{\prime}\right) M\left(v^{\prime}\right) f(x, v) \mathrm{d} v^{\prime}\right\rangle=\omega_{c}^{2} \int_{\mathbb{R}^{2}} \int_{\mathbb{R}^{3}} \mathcal{S}\left(|\bar{v}|, v_{3},\left|\overline{v^{\prime}}\right|, v_{3}^{\prime}, z\right) M\left(v^{\prime}\right) f(x, v) \mathrm{d} v^{\prime} \mathrm{d} x_{1}^{\prime} \mathrm{d} x_{2}^{\prime}
$$

and our statement follows immediately.

We intend to extend the previous averaged collision operator to all densities $f$, not only those satisfying the constraint $\mathcal{T} f=0$. Think that, when simulating numerically these models, the particle density may not satisfy exactly $\mathcal{T} f=0$, and thus we need to construct such a extension. One possibility consists to appeal to the decomposition $f=\langle f\rangle+(f-\langle f\rangle)$ and to neglect the fluctuation $f-\langle f\rangle$, leading to the operator

$$
\begin{aligned}
f \rightarrow & \tilde{Q}_{B} f:=\left\langle Q_{B}\langle f\rangle\right\rangle \\
& =\frac{\omega_{c}^{2}}{\tau} \int_{\mathbb{R}^{2}} \int_{\mathbb{R}^{3}} \mathcal{S}\left(|\bar{v}|, v_{3},\left|\overline{v^{\prime}}\right|, v_{3}^{\prime}, z\right)\left\{M(v)\langle f\rangle\left(\overline{x^{\prime}}, x_{3}, v^{\prime}\right)-M\left(v^{\prime}\right)\langle f\rangle(x, v)\right\} \mathrm{d} v^{\prime} \mathrm{d} x_{1}^{\prime} \mathrm{d} x_{2}^{\prime}
\end{aligned}
$$

for any $f \in L^{2}\left(M^{-1} \mathrm{~d} x \mathrm{~d} v\right)$. Clearly $\tilde{Q}_{B}$ coincides with $\left\langle Q_{B} f\right\rangle$ for any $f \in \operatorname{ker} \mathcal{T}$. Notice that for any $(x, v),\left(x_{3}^{\prime}, v_{3}^{\prime}\right)$ the function

$$
\left(\overline{x^{\prime}}, \overline{v^{\prime}}\right) \rightarrow \mathcal{S}\left(|\bar{v}|, v_{3},\left|\overline{v^{\prime}}\right|, v_{3}^{\prime}, \omega_{c} \bar{x}+{ }^{\perp} \bar{v}-\left(\omega_{c} \overline{x^{\prime}}+{ }^{\perp} \overline{v^{\prime}}\right)\right) M(v)
$$

depends only on $\omega_{c} \overline{x^{\prime}}+{ }^{\perp} \overline{v^{\prime}},\left|\overline{v^{\prime}}\right|$ and therefore, thanks to Remark 2.1, we obtain a simpler form

$$
\tilde{Q}_{B} f=\frac{\omega_{c}^{2}}{\tau} \int_{\mathbb{R}^{2}} \int_{\mathbb{R}^{3}} \mathcal{S}\left(|\bar{v}|, v_{3},\left|\overline{v^{\prime}}\right|, v_{3}^{\prime}, z\right)\left\{M(v) f\left(\overline{x^{\prime}}, x_{3}, v^{\prime}\right)-M\left(v^{\prime}\right)\langle f\rangle(x, v)\right\} \mathrm{d} v^{\prime} \mathrm{d} x_{1}^{\prime} \mathrm{d} x_{2}^{\prime} .
$$


Nevertheless notice that it is not possible to remove the average in the loss part of the previous operator. Since $Q_{B}$ and $\langle\cdot\rangle$ are linear bounded operators on $L^{2}\left(M^{-1} \mathrm{~d} x \mathrm{~d} v\right)$ we deduce that $\tilde{Q}_{B}$ is linear bounded on $L^{2}\left(M^{-1} \mathrm{~d} x \mathrm{~d} v\right)$. The properties of $\tilde{Q}_{B}$ come immediately from the properties of $Q_{B}$, cf. Proposition 4.1. For example we have for any $f \in L^{2}\left(M^{-1} \mathrm{~d} x \mathrm{~d} v\right)$

$$
\begin{aligned}
\int_{\mathbb{R}^{3}} \int_{\mathbb{R}^{3}} \tilde{Q}_{B} f \frac{f}{M} \mathrm{~d} v \mathrm{~d} x=\int_{\mathbb{R}^{3}} \int_{\mathbb{R}^{3}}\left\langle Q_{B}\langle f\rangle\right\rangle \frac{f}{M} \mathrm{~d} v \mathrm{~d} x=\int_{\mathbb{R}^{3}} \int_{\mathbb{R}^{3}} Q_{B}\langle f\rangle \frac{\langle f\rangle}{M} \mathrm{~d} v \mathrm{~d} x \\
=-\frac{1}{2 \tau} \int_{\mathbb{R}^{3}} \int_{\mathbb{R}^{3}} \int_{\mathbb{R}^{3}} s\left(v, v^{\prime}\right) M(v) M\left(v^{\prime}\right)\left[\frac{\langle f\rangle(x, v)}{M(v)}-\frac{\langle f\rangle\left(x, v^{\prime}\right)}{M\left(v^{\prime}\right)}\right]^{2} \mathrm{~d} v^{\prime} \mathrm{d} v \mathrm{~d} x \leq 0 .
\end{aligned}
$$

Another possible extension is given by

$$
\left\langle Q_{B}\right\rangle f:=\frac{\omega_{c}^{2}}{\tau} \int_{\mathbb{R}^{2}} \int_{\mathbb{R}^{3}} \mathcal{S}\left(|\bar{v}|, v_{3},\left|\overline{v^{\prime}}\right|, v_{3}^{\prime}, z\right)\left\{M(v) f\left(\overline{x^{\prime}}, x_{3}, v^{\prime}\right)-M\left(v^{\prime}\right) f(x, v)\right\} \mathrm{d} v^{\prime} \mathrm{d} x_{1}^{\prime} \mathrm{d} x_{2}^{\prime}
$$

for any $f \in L^{2}\left(M^{-1} \mathrm{~d} x \mathrm{~d} v\right)$, which is very similar to the operator $Q_{B}$ in (4.1). We keep this operator as extension for the operator in Corollary 4.1. The properties of $\left\langle Q_{B}\right\rangle$ are summarized below

Proposition 4.3. Assume that the scattering cross section satisfies (4.2), (4.7). Then 1. The operator $\left\langle Q_{B}\right\rangle$ is linear bounded on $L^{2}\left(M^{-1} \mathrm{~d} x \mathrm{~d} v\right)$ and symmetric with respect to the scalar product of $L^{2}\left(M^{-1} \mathrm{~d} x \mathrm{~d} v\right)$.

2. For any $f \in L^{2}\left(M^{-1} \mathrm{~d} x \mathrm{~d} v\right)$ we have

$$
\begin{aligned}
\int_{\mathbb{R}^{3}} \int_{\mathbb{R}^{3}}\left\langle Q_{B}\right\rangle(f) \frac{f}{M} \mathrm{~d} v \mathrm{~d} x & =-\frac{\omega_{c}^{2}}{2 \tau} \int_{\mathbb{R}^{3}} \int_{\mathbb{R}^{3}} \int_{\mathbb{R}^{2}} \int_{\mathbb{R}^{3}} \mathcal{S}\left(|\bar{v}|, v_{3},\left|\overline{v^{\prime}}\right|, v_{3}^{\prime}, z\right) M(v) M\left(v^{\prime}\right) \\
& \times\left[\frac{f(x, v)}{M(v)}-\frac{f\left(\overline{x^{\prime}}, x_{3}, v^{\prime}\right)}{M\left(v^{\prime}\right)}\right]^{2} \mathrm{~d} v^{\prime} \mathrm{d} x_{1}^{\prime} \mathrm{d} x_{2}^{\prime} \mathrm{d} v \mathrm{~d} x \leq 0
\end{aligned}
$$

Proof. 1. The boundedness of the loss part follows easily since it is the multiplication by the bounded function (see Remark 4.3)

$$
\begin{aligned}
\frac{\omega_{c}^{2}}{\tau} \int_{\mathbb{R}^{2}} \int_{\mathbb{R}^{3}} \mathcal{S}\left(|\bar{v}|, v_{3},\left|\overline{v^{\prime}}\right|, v_{3}^{\prime}, z\right) M\left(v^{\prime}\right) \mathrm{d} v^{\prime} \mathrm{d} x_{1}^{\prime} \mathrm{d} x_{2}^{\prime} & =\frac{1}{\tau} \int_{\mathbb{R}^{3}} \int_{\mathbb{R}^{2}} \mathcal{S}\left(|\bar{v}|, v_{3},\left|\overline{v^{\prime}}\right|, v_{3}^{\prime},-z^{\prime}\right) M\left(v^{\prime}\right) \mathrm{d} z^{\prime} \mathrm{d} v^{\prime} \\
& \leq \frac{S_{0}}{\tau} .
\end{aligned}
$$


For the gain part we use the inequalities

$$
\begin{aligned}
& \omega_{c}^{2}\left(\int_{\mathbb{R}^{2}} \int_{\mathbb{R}^{3}} \mathcal{S}\left(|\bar{v}|, v_{3},\left|\overline{v^{\prime}}\right|, v_{3}^{\prime}, z\right) f\left(\overline{x^{\prime}}, x_{3}, v^{\prime}\right) \mathrm{d} v^{\prime} \mathrm{d} x_{1}^{\prime} \mathrm{d} x_{2}^{\prime}\right)^{2} \\
& \leq \int_{\mathbb{R}^{2}} \int_{\mathbb{R}^{3}} \mathcal{S} \frac{f^{2}\left(\overline{x^{\prime}}, x_{3}, v^{\prime}\right)}{M\left(v^{\prime}\right)} \mathrm{d} v^{\prime} \mathrm{d} x_{1}^{\prime} \mathrm{d} x_{2}^{\prime} \omega_{c}^{2} \int_{\mathbb{R}^{2}} \int_{\mathbb{R}^{3}} \mathcal{S} M\left(v^{\prime}\right) \mathrm{d} v^{\prime} \mathrm{d} x_{1}^{\prime} \mathrm{d} x_{2}^{\prime} \\
& \leq S_{0} \int_{\mathbb{R}^{2}} \int_{\mathbb{R}^{3}} \mathcal{S} \frac{f^{2}\left(\overline{x^{\prime}}, x_{3}, v^{\prime}\right)}{M\left(v^{\prime}\right)} \mathrm{d} v^{\prime} \mathrm{d} x_{1}^{\prime} \mathrm{d} x_{2}^{\prime} .
\end{aligned}
$$

Thanks to Remark 4.3 we deduce, with $L_{M}^{2}=L^{2}\left(M^{-1} \mathrm{~d} x \mathrm{~d} v\right)$, that

$$
\begin{aligned}
& \left\|\frac{\omega_{c}^{2}}{\tau} \int_{\mathbb{R}^{2}} \int_{\mathbb{R}^{3}} \mathcal{S} M(v) f\left(\overline{x^{\prime}}, x_{3}, v^{\prime}\right) \mathrm{d} v^{\prime} \mathrm{d} x_{1}^{\prime} \mathrm{d} x_{2}^{\prime}\right\|_{L_{M}^{2}}^{2} \leq \frac{\omega_{c}^{2}}{\tau^{2}} \int_{\mathbb{R}^{3}} \int_{\mathbb{R}^{3}} M(v) S_{0} \\
& \times \int_{\mathbb{R}^{2}} \int_{\mathbb{R}^{3}} \mathcal{S} \frac{f^{2}\left(\overline{x^{\prime}}, x_{3}, v^{\prime}\right)}{M\left(v^{\prime}\right)} \mathrm{d} v^{\prime} \mathrm{d} x_{1}^{\prime} \mathrm{d} x_{2}^{\prime} \mathrm{d} v \mathrm{~d} x \\
& =\frac{S_{0}}{\tau^{2}} \int_{\mathbb{R}^{3}} M(v) \int_{\mathbb{R}^{3}} \int_{\mathbb{R}^{3}} \frac{f^{2}\left(\overline{x^{\prime}}, x_{3}, v^{\prime}\right)}{M\left(v^{\prime}\right)} \omega_{c}^{2} \int_{\mathbb{R}^{2}} \mathcal{S} \mathrm{d} x_{1} \mathrm{~d} x_{2} \mathrm{~d} v^{\prime} \mathrm{d} x_{1}^{\prime} \mathrm{d} x_{2}^{\prime} \mathrm{d} x_{3} \mathrm{~d} v \\
& \leq \frac{S_{0}^{2}}{\tau^{2}} \int_{\mathbb{R}^{3}} \int_{\mathbb{R}^{3}} \frac{f^{2}\left(x_{1}^{\prime}, x_{2}^{\prime}, x_{3}, v^{\prime}\right)}{M\left(v^{\prime}\right)} \mathrm{d} v^{\prime} \mathrm{d} x_{1}^{\prime} \mathrm{d} x_{2}^{\prime} \mathrm{d} x_{3}=\frac{S_{0}^{2}}{\tau^{2}}\|f\|_{L_{M}^{2}}^{2} .
\end{aligned}
$$

2. Interchanging $\left(\overline{x^{\prime}}, v^{\prime}\right)$ with $(\bar{x}, v)$ and observing that this change leaves invariant $\mathcal{S}$, yield for any $f, g \in L^{2}\left(M^{-1} \mathrm{~d} x \mathrm{~d} v\right)$

$$
\begin{aligned}
& \left(\left\langle Q_{B}\right\rangle f, g\right)_{L_{M}^{2}} \\
& =\frac{\omega_{c}^{2}}{\tau} \int_{\mathbb{R}^{3}} \int_{\mathbb{R}^{3}} \int_{\mathbb{R}^{2}} \int_{\mathbb{R}^{3}} \mathcal{S} M(v) M\left(v^{\prime}\right)\left[\frac{f\left(\overline{x^{\prime}}, x_{3}, v^{\prime}\right)}{M\left(v^{\prime}\right)}-\frac{f(x, v)}{M(v)}\right] \mathrm{d} v^{\prime} \mathrm{d} x_{1}^{\prime} \mathrm{d} x_{2}^{\prime} \frac{g(x, v)}{M(v)} \mathrm{d} v \mathrm{~d} x \\
& =-\frac{\omega_{c}^{2}}{\tau} \int_{\mathbb{R}^{3}} \int_{\mathbb{R}^{3}} \int_{\mathbb{R}^{2}} \int_{\mathbb{R}^{3}} \mathcal{S} M(v) M\left(v^{\prime}\right)\left[\frac{f\left(\overline{x^{\prime}}, x_{3}, v^{\prime}\right)}{M\left(v^{\prime}\right)}-\frac{f(x, v)}{M(v)}\right] \mathrm{d} v \mathrm{~d} x_{1} \mathrm{~d} x_{2} \\
& \times \frac{g\left(\overline{x^{\prime}}, x_{3}, v^{\prime}\right)}{M\left(v^{\prime}\right)} \mathrm{d} v^{\prime} \mathrm{d} x_{1}^{\prime} \mathrm{d} x_{2}^{\prime} \mathrm{d} x_{3} \\
& =-\frac{\omega_{c}^{2}}{2 \tau} \int_{\mathbb{R}^{3}} \int_{\mathbb{R}^{3}} \int_{\mathbb{R}^{2}} \int_{\mathbb{R}^{3}} \mathcal{S} M(v) M\left(v^{\prime}\right)\left[\frac{f\left(\overline{x^{\prime}}, x_{3}, v^{\prime}\right)}{M\left(v^{\prime}\right)}-\frac{f(x, v)}{M(v)}\right]\left[\frac{g\left(\overline{x^{\prime}}, x_{3}, v^{\prime}\right)}{M\left(v^{\prime}\right)}-\frac{g(x, v)}{M(v)}\right] \\
& \mathrm{d} v^{\prime} \mathrm{d} x_{1}^{\prime} \mathrm{d} x_{2}^{\prime} \mathrm{d} v \mathrm{~d} x
\end{aligned}
$$

which justifies the symmetry of $\left\langle Q_{B}\right\rangle$ and its negativity. 
REmark 4.5. Contrary to $Q_{B}$, the operator $\left\langle Q_{B}\right\rangle$ is non local in space. The value of $\left\langle Q_{B}\right\rangle f$ at the point $(x, v)$ depends on all the values of $f$ in the set

$$
\begin{aligned}
A(x, v) & =\left\{\left(x_{1}^{\prime}, x_{2}^{\prime}, x_{3}, v^{\prime}\right): \mathcal{S}\left(|\bar{v}|, v_{3},\left|\overline{v^{\prime}}\right|, v_{3}^{\prime},\left(\omega_{c} \bar{x}+{ }^{\perp} \bar{v}\right)-\left(\omega_{c} \overline{x^{\prime}}+{ }^{\perp} \overline{v^{\prime}}\right)\right)>0\right\} \\
& =\left\{\left(x_{1}^{\prime}, x_{2}^{\prime}, x_{3}, v^{\prime}\right):|| \bar{v}|-| \overline{v^{\prime}}||<\left|\left(\omega_{c} \bar{x}+{ }^{\perp} \bar{v}\right)-\left(\omega_{c} \overline{x^{\prime}}+{ }^{\perp} \overline{v^{\prime}}\right)\right|<|\bar{v}|+\left|\overline{v^{\prime}}\right|\right\} .
\end{aligned}
$$

Observe that if we denote by $C_{x, v}$ the Larmor circle

$$
C_{x, v}=\left\{\left(x_{1}^{\prime}, x_{2}^{\prime}, x_{3}\right):\left|\omega_{c} \overline{x^{\prime}}-\left(\omega_{c} \bar{x}+{ }^{\perp} \bar{v}\right)\right|=|\bar{v}|\right\}
$$

then we have

$$
C_{x, v} \times\left\{v^{\prime}: v^{\prime} \in \mathbb{R}^{3}\right\} \subset \overline{A(x, v)}
$$

where $\bar{X}$ stands for the adherence of $X$ in $\mathbb{R}^{6}$. In particular $\{x\} \times \mathbb{R}^{3} \subset \overline{A(x, v)}$.

REMARK 4.6. The gain/loss parts of $\left\langle Q_{B}\right\rangle$ are bounded on $L^{1}\left(\mathbb{R}^{3} \times \mathbb{R}^{3}\right)$ and for any $f \in L^{1}\left(\mathbb{R}^{3} \times \mathbb{R}^{3}\right)$ we have the global balance of the mass $\int_{\mathbb{R}^{3}} \int_{\mathbb{R}^{3}}\left\langle Q_{B}\right\rangle f \mathrm{~d} v \mathrm{~d} x=0$. Indeed, we have

$$
\begin{aligned}
\left\|\left\langle Q_{B}\right\rangle^{+} f\right\|_{L^{1}} & \leq \frac{\omega_{c}^{2}}{\tau} \int_{\mathbb{R}^{3}} \int_{\mathbb{R}^{3}} \int_{\mathbb{R}^{2}} \int_{\mathbb{R}^{3}} \mathcal{S}\left(|\bar{v}|, v_{3},\left|\overline{v^{\prime}}\right|, v_{3}^{\prime}, z\right) M(v)\left|f\left(\overline{x^{\prime}}, x_{3}, v^{\prime}\right)\right| \mathrm{d} v^{\prime} \mathrm{d} x_{1}^{\prime} \mathrm{d} x_{2}^{\prime} \mathrm{d} v \mathrm{~d} x \\
& \leq \frac{S_{0}}{\tau} \int_{\mathbb{R}^{3}} \int_{\mathbb{R}^{3}} \int_{\mathbb{R}^{3}} M(v)\left|f\left(\overline{x^{\prime}}, x_{3}, v^{\prime}\right)\right| \mathrm{d} v^{\prime} \mathrm{d} x_{1}^{\prime} \mathrm{d} x_{2}^{\prime} \mathrm{d} x_{3} \mathrm{~d} v \\
& =\frac{S_{0}}{\tau}\|f\|_{L^{1}}
\end{aligned}
$$

and similarly

$$
\left\|\left\langle Q_{B}\right\rangle^{-} f\right\|_{L^{1}} \leq \frac{S_{0}}{\tau}\|f\|_{L^{1}}
$$

The global balance follows by interchanging $\left(\overline{x^{\prime}}, v^{\prime}\right)$ with $(\bar{x}, v)$.

Combining (1.6), Propositions 3.4, 4.1 and (4.8) yields the limit model in Theorem 1.1.

Proof. (of Theorem 1.1) Clearly $0 \leq f^{\varepsilon} \in L^{\infty}\left(\mathbb{R}_{+}, L^{1}\left(\mathbb{R}^{3} \times \mathbb{R}^{3}\right)\right.$ ) and

$$
\int_{\mathbb{R}^{3}} \int_{\mathbb{R}^{3}} f^{\varepsilon}(t, x, v) \mathrm{d} v \mathrm{~d} x=\int_{\mathbb{R}^{3}} \int_{\mathbb{R}^{3}} f^{\mathrm{in}}(x, v) \mathrm{d} v \mathrm{~d} x, \quad t \in \mathbb{R}_{+}, \varepsilon>0 .
$$

We consider a sequence $\left(\varepsilon_{k}\right)_{k} \subset \mathbb{R}_{+}^{\star}$ converging to 0 such that $\lim _{k \rightarrow+\infty} f^{\varepsilon_{k}}=f$ weakly $\star$ in $L^{\infty}\left(\mathbb{R}_{+}, L^{2}\left(M^{-1} \mathrm{~d} x \mathrm{~d} v\right)\right)$. Using the weak formulation of (1.3), (1.2) with test 
functions $\eta(t) \varphi(x, v), \eta \in C_{c}^{1}\left(\mathbb{R}_{+}\right), \varphi \in C_{c}^{1}\left(\mathbb{R}^{3} \times \mathbb{R}^{3}\right)$, we deduce, after multiplication by $\varepsilon_{k}$ and letting $k \rightarrow \infty$, that the limit density satisfies the constraint

$$
\mathcal{T} f(t)=0, \quad t \in \mathbb{R}_{+} .
$$

Considering test functions like $\eta(t) \varphi(x, v)$ with $\eta \in C_{c}^{1}\left(\mathbb{R}_{+}\right), \varphi \in C_{c}^{1}\left(\mathbb{R}^{3} \times \mathbb{R}^{3}\right) \cap \operatorname{ker} \mathcal{T}$ one gets

$$
\begin{aligned}
\int_{\mathbb{R}_{+}} \int_{\mathbb{R}^{3}} \int_{\mathbb{R}^{3}} f^{\varepsilon_{k}}\left\{\eta^{\prime} \varphi+\eta v_{3} \partial_{x_{3}} \varphi+\eta \frac{q}{m} E(x) \cdot \nabla_{v} \varphi\right\} \mathrm{d} v \mathrm{~d} x \mathrm{~d} t & +\int_{\mathbb{R}^{3}} \int_{\mathbb{R}^{3}} f^{\mathrm{in}} \eta(0) \varphi \mathrm{d} v \mathrm{~d} x \\
& =-\int_{\mathbb{R}_{+}} \int_{\mathbb{R}^{3}} \int_{\mathbb{R}^{3}} \eta Q_{B}\left(f^{\varepsilon_{k}}\right) \varphi \mathrm{d} v \mathrm{~d} x \mathrm{~d} t .
\end{aligned}
$$

The symmetry of $Q_{B}$ cf. Proposition 4.1 allows us to write

$$
\begin{aligned}
\lim _{k \rightarrow+\infty} \int_{\mathbb{R}_{+}} \int_{\mathbb{R}^{3}} \int_{\mathbb{R}^{3}} \eta Q_{B}\left(f^{\varepsilon_{k}}\right) \varphi \mathrm{d} v \mathrm{~d} x \mathrm{~d} t=\lim _{k \rightarrow+\infty} \int_{\mathbb{R}_{+}} \int_{\mathbb{R}^{3}} \int_{\mathbb{R}^{3}} \eta f^{\varepsilon_{k}} Q_{B}(\varphi M) \frac{\mathrm{d} v \mathrm{~d} x}{M} \mathrm{~d} t \\
=\int_{\mathbb{R}_{+}} \int_{\mathbb{R}^{3}} \int_{\mathbb{R}^{3}} \eta f Q_{B}(\varphi M) \frac{\mathrm{d} v \mathrm{~d} x}{M} \mathrm{~d} t=\int_{\mathbb{R}_{+}} \int_{\mathbb{R}^{3}} \int_{\mathbb{R}^{3}} \eta Q_{B}(f) \varphi \mathrm{d} v \mathrm{~d} x \mathrm{~d} t \\
=\int_{\mathbb{R}_{+}} \int_{\mathbb{R}^{3}} \int_{\mathbb{R}^{3}} \eta\left\langle Q_{B}(f)\right\rangle \varphi \mathrm{d} v \mathrm{~d} x \mathrm{~d} t=\int_{\mathbb{R}_{+}} \int_{\mathbb{R}^{3}} \int_{\mathbb{R}^{3}} \eta\left\langle Q_{B}\right\rangle(f) \varphi \mathrm{d} v \mathrm{~d} x \mathrm{~d} t
\end{aligned}
$$

since $f(t) \in \operatorname{ker} \mathcal{T}, t \in \mathbb{R}_{+}$and thus $\left\langle Q_{B}(f)\right\rangle=\left\langle Q_{B}\right\rangle(f)$. For the other terms in (4.10) we obtain thanks to Proposition 3.4, Remark 3.2

$$
\begin{aligned}
\lim _{k \rightarrow+\infty} \int_{\mathbb{R}_{+}} \int_{\mathbb{R}^{3}} \int_{\mathbb{R}^{3}} f^{\varepsilon_{k}}\left(\partial_{t}+a \cdot \nabla_{x, v}\right)(\eta \varphi) \mathrm{d} v \mathrm{~d} x \mathrm{~d} t & =\int_{\mathbb{R}_{+}} \int_{\mathbb{R}^{3}} \int_{\mathbb{R}^{3}} f\left(\partial_{t}+a \cdot \nabla_{x, v}\right)(\eta \varphi) \mathrm{d} v \mathrm{~d} x \mathrm{~d} t \\
& =\int_{\mathbb{R}_{+}} \int_{\mathbb{R}^{3}} \int_{\mathbb{R}^{3}} f\left(\partial_{t}+A \cdot \nabla_{x, v}\right)(\eta \varphi) \mathrm{d} v \mathrm{~d} x \mathrm{~d} t
\end{aligned}
$$

and

$$
\int_{\mathbb{R}^{3}} \int_{\mathbb{R}^{3}} f^{\mathrm{in}}(x, v) \eta(0) \varphi(x, v) \mathrm{d} v \mathrm{~d} x=\int_{\mathbb{R}^{3}} \int_{\mathbb{R}^{3}} \eta(0)\left\langle f^{\mathrm{in}}\right\rangle(x, v) \varphi(x, v) \mathrm{d} v \mathrm{~d} x .
$$


Combining (4.10), (4.11), (4.12), (4.13) yields for any smooth test function $\eta(t) \varphi(x, v)$ with $\varphi \in \operatorname{ker} \mathcal{T}$.

$$
\begin{aligned}
\int_{\mathbb{R}_{+}} \int_{\mathbb{R}^{3}} \int_{\mathbb{R}^{3}} f\left(\partial_{t}+A \cdot \nabla_{x, v}\right)(\eta \varphi) \mathrm{d} v \mathrm{~d} x \mathrm{~d} t & +\int_{\mathbb{R}^{3}} \int_{\mathbb{R}^{3}}\left\langle f^{\mathrm{in}}\right\rangle \eta(0) \varphi(x, v) \mathrm{d} v \mathrm{~d} x \\
& =-\int_{\mathbb{R}_{+}} \int_{\mathbb{R}^{3}} \int_{\mathbb{R}^{3}}\left\langle Q_{B}\right\rangle(f) \eta \varphi \mathrm{d} v \mathrm{~d} x \mathrm{~d} t .
\end{aligned}
$$

By Remark 3.2 we know that $A \cdot \nabla_{x, v}$ leaves invariant the subspace of zero average functions and therefore it is easily seen that (4.14) is trivially satisfied for any test function $\eta(t) \psi(x, v)$, with $\psi \in C_{c}^{1}\left(\mathbb{R}^{3} \times \mathbb{R}^{3}\right) \cap \operatorname{ker}\langle\cdot\rangle$. Finally (4.14) holds true for any smooth test function, saying that $f$ solves (1.9), (1.10). We are done if we prove the uniqueness for the solution of (1.9), (1.10) (and in this case all the family $\left(f^{\varepsilon}\right)_{\varepsilon}$ will converge towards $f$, weakly $\star$ in $\left.L^{\infty}\left(\mathbb{R}_{+}, L^{2}\left(M^{-1} \mathrm{~d} x \mathrm{~d} v\right)\right)\right)$. Assume that $f$ solves (1.9) with zero initial condition. By standard arguments one gets

$$
\partial_{t}|f|+\frac{\langle\perp \bar{E}\rangle}{B} \cdot \nabla_{\bar{x}}|f|+v_{3} \partial_{x_{3}}|f|+\frac{q}{m}\left\langle E_{3}\right\rangle \partial_{v_{3}}|f|=\left\langle Q_{B}\right\rangle(f) \operatorname{sgn} f
$$

implying that

$$
\frac{\mathrm{d}}{\mathrm{d} t} \int_{\mathbb{R}^{3}} \int_{\mathbb{R}^{3}}|f(t, x, v)| \mathrm{d} v \mathrm{~d} x=\int_{\mathbb{R}^{3}} \int_{\mathbb{R}^{3}}\left\langle Q_{B}\right\rangle(f) \operatorname{sgn} f(t, x, v) \mathrm{d} v \mathrm{~d} x, \quad t \in \mathbb{R}_{+} .
$$

Our conclusion comes by observing that

$$
\begin{aligned}
& \int_{\mathbb{R}^{3}} \int_{\mathbb{R}^{3}}\left\langle Q_{B}\right\rangle(f) \operatorname{sgn} f \mathrm{~d} v \mathrm{~d} x \\
& =\frac{\omega_{c}^{2}}{\tau} \int_{\mathbb{R}^{3}} \int_{\mathbb{R}^{3}} \int_{\mathbb{R}^{2}} \int_{\mathbb{R}^{3}} \mathcal{S}\left\{M(v) f\left(t, \overline{x^{\prime}}, x_{3}, v^{\prime}\right)-M\left(v^{\prime}\right) f(t, x, v)\right\} \operatorname{sgn} f(t, x, v) \mathrm{d} v^{\prime} \mathrm{d} x_{1}^{\prime} \mathrm{d} x_{2}^{\prime} \mathrm{d} v \mathrm{~d} x \\
& =\frac{\omega_{c}^{2}}{\tau} \int_{\mathbb{R}^{3}} \int_{\mathbb{R}^{3}} \int_{\mathbb{R}^{2}} \int_{\mathbb{R}^{3}} \mathcal{S} M\left(v^{\prime}\right)\left\{f(t, x, v) \operatorname{sgn} f\left(t, \overline{x^{\prime}}, x_{3}, v^{\prime}\right)-|f(t, x, v)|\right\} \mathrm{d} v^{\prime} \mathrm{d} x_{1}^{\prime} \mathrm{d} x_{2}^{\prime} \mathrm{d} v \mathrm{~d} x \leq 0 .
\end{aligned}
$$

REMARK 4.7. It is easily seen that the integro-differential operator in (1.9) propagates the constraint $\mathcal{T} f=0$. We are done if we prove that $f_{s}=f$ for any $s \in \mathbb{R}$, where $f_{s}(t, x, v)=f(t, X(s ; x, v), V(s ; x, v))$ and $(X, V)$ is the characteristic flow (1.7). A direct computation shows that $\mathcal{T}$ and $A \cdot \nabla_{x, v}=\frac{\left\langle{ }^{\perp} \bar{E}\right\rangle}{B} \cdot \nabla_{\bar{x}}+v_{3} \partial_{x_{3}}+\frac{q}{m}\left\langle E_{3}\right\rangle \partial_{v_{3}}$ commute, implying that

$$
A \cdot \nabla_{x, v} f_{s}=\left(A \cdot \nabla_{x, v} f\right)_{s} .
$$


Observe also that

$$
\left\langle Q_{B}\right\rangle^{+} f_{s}=\left\langle Q_{B}\right\rangle^{+} f=\left(\left\langle Q_{B}\right\rangle^{+} f\right)_{s},\left\langle Q_{B}\right\rangle^{-} f_{s}=\left(\left\langle Q_{B}\right\rangle^{-} f\right)_{s}
$$

and therefore $\left\langle Q_{B}\right\rangle f_{s}=\left(\left\langle Q_{B}\right\rangle f\right)_{s}$. Finally both $f, f_{s}$ satisfy (1.9), (1.10) and our statement follows by the uniqueness that we have established before.

Clearly the transport equation $(1.9)$ can be written in the reduced phase space $(\bar{y}=$ $\left.\bar{x}+\frac{{ }^{\perp} \bar{v}}{\omega_{c}}, x_{3}, r=|\bar{v}|, v_{3}\right)$ since, by the constraint $\mathcal{T} f=0$, we know that $f(t, x, v)=$ $g\left(t, \bar{y}, x_{3}, r, v_{3}\right)$. We obtain

$$
\begin{aligned}
\partial_{t} g+\frac{\left\langle{ }^{\perp} \bar{E}\right\rangle}{B} \cdot \nabla_{\bar{y}} g+v_{3} \partial_{x_{3}} g & +\frac{q}{m}\left\langle E_{3}\right\rangle \partial_{v_{3}} g=2 \pi \frac{\omega_{c}^{2}}{\tau} \int_{\mathbb{R}^{2}} \int_{\mathbb{R}} \int_{\mathbb{R}_{+}} \mathcal{S}\left(r, v_{3}, r^{\prime}, v_{3}^{\prime}, \omega_{c}\left(\bar{y}-\overline{y^{\prime}}\right)\right) \\
\times & \left\{M g\left(t, \overline{y^{\prime}}, x_{3}, r^{\prime}, v_{3}^{\prime}\right)-M^{\prime} g\left(t, \bar{y}, x_{3}, r, v_{3}\right)\right\} r^{\prime} \mathrm{d} r^{\prime} \mathrm{d} v_{3}^{\prime} \mathrm{d} \overline{y^{\prime}}
\end{aligned}
$$

where

$$
M=\frac{1}{(2 \pi \theta / m)^{3 / 2}} e^{-\frac{m}{2 \theta}\left(r^{2}+\left(v_{3}\right)^{2}\right)}, \quad M^{\prime}=\frac{1}{(2 \pi \theta / m)^{3 / 2}} e^{-\frac{m}{2 \theta}\left(\left(r^{\prime}\right)^{2}+\left(v_{3}^{\prime}\right)^{2}\right)} .
$$

REMARK 4.8. The family $\left(f^{\varepsilon}\right)_{0<\varepsilon \leq 1}$ remains bounded in $L^{\infty}\left(\mathbb{R}_{+}, L^{2}\left(M^{-1} \mathrm{~d} x \mathrm{~d} v\right)\right)$ for potentials of the form $\phi(x)=\bar{\phi}(\bar{x})+\phi_{3}\left(x_{3}\right)$. Indeed, in this case observe that the energy function $W^{\varepsilon}(x, v):=\frac{m|v|^{2}}{2}+q\left(\varepsilon \bar{\phi}(\bar{x})+\phi_{3}\left(x_{3}\right)\right)$ satisfies

$$
\partial_{t} W^{\varepsilon}+\frac{1}{\varepsilon}\left(\bar{v} \cdot \nabla_{\bar{x}}+\omega_{c}{ }^{\perp} \bar{v} \cdot \nabla_{\bar{v}}\right) W^{\varepsilon}+v_{3} \partial_{x_{3}} W^{\varepsilon}+\frac{q}{m} E(x) \cdot \nabla_{v} W^{\varepsilon}=0
$$

and therefore one gets

$$
\begin{aligned}
\left\{\partial_{t}+\frac{1}{\varepsilon}\left(\bar{v} \cdot \nabla_{\bar{x}}+\omega_{c}{ }^{\perp} \bar{v} \cdot \nabla_{\bar{v}}\right)+v_{3} \partial_{x_{3}}+\frac{q}{m} E(x) \cdot \nabla_{v}\right\} & \left(\frac{\left(f^{\varepsilon}\right)^{2}}{2 M(v) \exp \left(-\frac{q}{\theta}\left[\varepsilon \bar{\phi}(\bar{x})+\phi_{3}\left(x_{3}\right)\right]\right)}\right) \\
& =\frac{Q_{B}\left(f^{\varepsilon}\right) f^{\varepsilon}}{M(v) \exp \left(-\frac{q}{\theta}\left[\varepsilon \bar{\phi}(\bar{x})+\phi_{3}\left(x_{3}\right)\right]\right)} .
\end{aligned}
$$

Integrating with respect to $(x, v)$ yields the bound

$\int_{\mathbb{R}^{3}} \int_{\mathbb{R}^{3}} \frac{\left(f^{\varepsilon}(t, x, v)\right)^{2}}{M(v) \exp \left(-\frac{q}{\theta}\left[\varepsilon \bar{\phi}(\bar{x})+\phi_{3}\left(x_{3}\right)\right]\right)} \mathrm{d} v \mathrm{~d} x \leq \int_{\mathbb{R}^{3}} \int_{\mathbb{R}^{3}} \frac{\left(f^{\text {in }}(x, v)\right)^{2}}{M(v) \exp \left(-\frac{q}{\theta}\left[\varepsilon \bar{\phi}(\bar{x})+\phi_{3}\left(x_{3}\right)\right]\right)} \mathrm{d} v \mathrm{~d} x$ implying the uniform estimate

$$
\left\|f^{\varepsilon}\right\|_{L^{\infty}\left(\mathbb{R}_{+}, L^{2}\left(M^{-1} \mathrm{~d} x \mathrm{~d} v\right)\right)} \leq \exp \left(\frac{|q|\left(\|\bar{\phi}\|_{L^{\infty}}+\left\|\phi_{3}\right\|_{\left.L^{\infty}\right)}\right.}{\theta}\right)\left\|f^{\mathrm{in}}\right\|_{L^{2}\left(M^{-1} \mathrm{~d} x \mathrm{~d} v\right)}, 0<\varepsilon \leq 1 .
$$




\section{Appendix A. Proof of Theorem 1.2.}

Proof. The Fokker-Planck kernel being a second order differential operator, we appeal twice to Proposition 3.3. For any $f \in \operatorname{ker} \mathcal{T}$, taking $\xi_{v}=M \nabla_{v}(f / M)$ yields

$$
\begin{aligned}
& \left\langle\operatorname{div}_{v}\left(M \nabla_{v}\left(\frac{f}{M}\right)\right)\right\rangle \\
& =\operatorname{div}_{\omega_{c} \bar{x}}\left\{\left\langle M^{\perp} \nabla_{\bar{v}}\left(\frac{f}{M}\right)\right\rangle+\left\langle M \nabla_{\bar{v}}\left(\frac{f}{M}\right) \cdot \frac{{ }^{\perp} \bar{v}}{|\bar{v}|}\right\rangle \frac{\bar{v}}{|\bar{v}|}-\left\langle M \nabla_{\bar{v}}\left(\frac{f}{M}\right) \cdot \frac{\bar{v}}{|\bar{v}|}\right\rangle \frac{{ }^{\perp} \bar{v}}{|\bar{v}|}\right\} \\
& +\operatorname{div}_{\bar{v}}\left\{\left\langle M \nabla_{\bar{v}}\left(\frac{f}{M}\right) \cdot \frac{{ }^{\perp} \bar{v}}{|\bar{v}|}\right\rangle \frac{{ }^{\perp} \bar{v}}{|\bar{v}|}+\left\langle M \nabla_{\bar{v}}\left(\frac{f}{M}\right) \cdot \frac{\bar{v}}{|\bar{v}|}\right\rangle \frac{\bar{v}}{|\bar{v}|}\right\}+\partial_{v_{3}}\left\langle M \partial_{v_{3}}\left(\frac{f}{M}\right)\right\rangle .
\end{aligned}
$$

Since $\partial_{v_{3}}$ commutes with $\langle\cdot\rangle$ (cf. Proposition 3.2) we deduce

$\partial_{v_{3}}\left\langle M \partial_{v_{3}}\left(\frac{f}{M}\right)\right\rangle=\partial_{v_{3}}\left\{M\left\langle\partial_{v_{3}}\left(\frac{f}{M}\right)\right\rangle\right\}=\partial_{v_{3}}\left\{M \partial_{v_{3}}\left\langle\frac{f}{M}\right\rangle\right\}=\partial_{v_{3}}\left\{M \partial_{v_{3}}\left(\frac{f}{M}\right)\right\}$.

It remains to compute the averages

$$
\left\langle M^{\perp} \nabla_{\bar{v}}\left(\frac{f}{M}\right)\right\rangle,\left\langle M \nabla_{\bar{v}}\left(\frac{f}{M}\right) \cdot \frac{\perp \bar{v}}{|\bar{v}|}\right\rangle,\left\langle M \nabla_{\bar{v}}\left(\frac{f}{M}\right) \cdot \frac{\bar{v}}{|\bar{v}|}\right\rangle .
$$

These averages come easily, thanks to Proposition 3.3, observing that

$$
\begin{aligned}
\partial_{v_{1}}\left(\frac{f}{M}\right) & =\operatorname{div}_{v}\left(\frac{f}{M}, 0,0\right), \partial_{v_{2}}\left(\frac{f}{M}\right)=\operatorname{div}_{v}\left(0, \frac{f}{M}, 0\right) \\
\nabla_{\bar{v}}\left(\frac{f}{M}\right) \cdot{ }^{\perp} \bar{v} & =\operatorname{div}_{\bar{v}}\left(\frac{f}{M} \perp \bar{v}\right), \quad \nabla_{\bar{v}}\left(\frac{f}{M}\right) \cdot \bar{v}=\operatorname{div}_{\bar{v}}\left(\frac{f}{M} \bar{v}\right)-2 \frac{f}{M} .
\end{aligned}
$$

We obtain

$$
\begin{gathered}
\left\langle M^{\perp} \nabla_{\bar{v}}\left(\frac{f}{M}\right)\right\rangle=M \nabla_{\omega_{c} \bar{x}}\left(\frac{f}{M}\right) \\
\left\langle M \nabla_{\bar{v}}\left(\frac{f}{M}\right) \cdot \frac{{ }^{\perp} \bar{v}}{|\bar{v}|}\right\rangle=M\left(\frac{(\bar{v}, 0)}{|\bar{v}|}, \frac{\left({ }^{\perp} \bar{v}, 0\right)}{|\bar{v}|}\right) \cdot \nabla_{\omega_{c} x, v}\left(\frac{f}{M}\right) \\
\left\langle M \nabla_{\bar{v}}\left(\frac{f}{M}\right) \cdot \frac{\bar{v}}{|\bar{v}|}\right\rangle=-M\left(\frac{\left({ }^{\perp} \bar{v}, 0\right)}{|\bar{v}|},-\frac{(\bar{v}, 0)}{|\bar{v}|}\right) \cdot \nabla_{\omega_{c} x, v}\left(\frac{f}{M}\right) .
\end{gathered}
$$

Our conclusion follows by combining (A.1), (A.2), (A.3), (A.4). The diffusion matrix $\mathcal{L}$ is positive and for any $\xi=\left(\xi_{x}, \xi_{v}\right) \in \mathbb{R}^{6}$ we have

$$
\mathcal{L} \xi \cdot \xi=\left|\xi_{\bar{x}}\right|^{2}+\left|\xi_{\bar{x}}-{ }^{\perp} \xi_{\bar{v}}\right|^{2}+\left(\xi_{v_{3}}\right)^{2} \geq 0
$$

with equality iff $\xi_{\bar{x}}=\xi_{\bar{v}}=(0,0)$ and $\xi_{v_{3}}=0$. 


\section{REFERENCES}

[1] M. Bostan, The Vlasov-Poisson system with strong external magnetic field. Finite Larmor radius regime, Asymptot. Anal. 61(2009) 91-123.

[2] M. Bostan, Transport equations with disparate advection fields. Application to the gyrokinetic models in plasma physics, J. Differential Equations 249(2010) 1620-1663.

[3] M. Bostan, Gyro-kinetic Vlasov equation in three dimensional setting. Second order approximation, SIAM J. Multiscale Model. Simul. 8(2010) 1923-1957.

[4] M. Bostan, Transport of charged particles under fast oscillating magnetic fields, SIAM J. Math. Anal. 44(2012) 1415-1447.

[5] M. Bostan, C. Caldini-Queiros, Finite Larmor radius approximation for collisional magnetized plasmas, C. R. Math. Acad. Sci. Paris Ser. I 350(2012) 879-884.

[6] M. Bostan, C. Caldini-Queiros, Finite Larmor radius approximation for collisional magnetic confinement. Part II : the Fokker-Planck-Landau equation, to appear in Quart. Appl. Math.

[7] A.J. Brizard, Variational principle for non linear gyrokinetic Vlasov-Maxwell equations, Phys. Plasmas 7(2000) 4816-4822.

[8] A.J. Brizard, A guiding-center Fokker-Planck collision operator for nonuniform magnetic fields, Phys. Plasmas 11(2004) 4429-4438.

[9] A.J. Brizard, T.S. Hahm, Foundations of nonlinear gyrokinetic theory, Rev. Modern Phys. 79(2007) 421-468.

[10] E. Frénod, Application of the averaging method to the gyrokinetic plasma, Asymptot. Anal. 46(2006) 1-28.

[11] E. Frénod, A. Mouton, Two-dimensional finite Larmor radius approximation in canonical gyrokinetic coordinates, J. Pures Appl. Math. Adv. Appl. 4(2010) 135-169.

[12] E. Frénod, E. Sonnendrücker, Homogenization of the Vlasov equation and of the Vlasov-Poisson system with strong external magnetic field, Asymptotic Anal. 18(1998) 193-213.

[13] E. Frénod, E. Sonnendrücker, The finite Larmor radius approximation, SIAM J. Math. Anal. $32(2001)$ 1227-1247.

[14] X. Garbet, G. Dif-Pradalier, C. Nguyen, Y. Sarazin, V. Grandgirard, Ph. Ghendrih, Neoclassical equilibrium in gyrokinetic simulations, Phys, Plasmas 16(2009).

[15] F. Golse, L. Saint-Raymond, The Vlasov-Poisson system with strong magnetic field, J. Math. Pures Appl. 78(1999) 791-817.

[16] P. Markowich, C. Ringhofer, C. Schmeiser, Semiconductor equations, Springer-Verlag, Vienna, 1990.

[17] F. Poupaud, C. Schmeiser, Charge transport in semiconductors with degeneracy effects, Math. Methods Appl. Sci. 14(1991) 301-318.

[18] F. Poupaud, Runaway phenomena and fluid approximation under high fields in semiconductor kinetic theory, Z. Angew. Math. Mech. 72(1992) 359-372. 
[19] L. Saint-Raymond, Control of large velocities in the two-dimensional gyrokinetic approximation, J. Math. Pures Appl. 81(2002) 379-399.

[20] X.Q. Xu, M.N. Rosenbluth, Numerical simulation of ion-temperature-gradient-driven modes, Phys. Fluids, B 3(1991) 627-643. 